\title{
GÖDEL ALGEBRAS: INTERACTIVE DUALITIES AND THEIR APPLICATIONS
}

\author{
L. M. CABRER AND H. A. PRIESTLEY
}

Dedicated to Brian Davey in celebration of his 65th birthday

\begin{abstract}
We present a technique for deriving certain new natural dualities for any variety of algebras generated by a finite Heyting chain. The dualities we construct are tailored to admit a transparent translation to the more pictorial Priestley/Esakia duality and back again. This enables us to combine the two approaches and so to capitalise on the virtues of both, in particular the categorical good behaviour of a natural duality: we thereby demonstrate the fullness, or not, of each of our dualities; we obtain new results on amalgamation; and we also provide a simple treatment of coproducts.
\end{abstract}

\section{INTRODUCTION}

This paper focuses on the classes of algebras $\mathcal{G}_{n}=\operatorname{ISP}\left(\mathbf{C}_{n}\right)$, where $\mathbf{C}_{n}$ is the $n$-element Heyting chain, and on natural dualities for them. As we recall below, the classes $\mathcal{G}_{n}$ are important both within and beyond duality theory.

It is highly appropriate that this topic should feature in the Special Issue of Algebra Universalis in honour of Brian Davey: through his work, the varieties $\mathcal{G}_{n}$ have been influential as a example within natural duality theory for nearly 40 years, beginning with his trail-blazing 1976 paper [7]. The advances to which study of these varieties have made a contribution are well chronicled, and fully referenced, by Davey and Talukder [9, Section 1]. Here we add a further chapter to the saga. Although the emphasis will be squarely on the varieties $\mathcal{G}_{n}$, which have very special features which work to our advantage, glimpses also open up of future avenues of investigation with wider scope.

Many varieties which provide illuminating test case examples for duality theory are also of relevance to logic. In particular any variety $\mathcal{A}$ of Heyting algebras is the algebraic counterpart of an intermediate logic $\mathcal{L}_{\mathcal{A}}$ (an axiomatic extension of intuitionistic propositional logic, IPC). The varieties $\mathcal{G}_{n}$ are the proper subvarieties of the variety $\mathcal{G}$ of Gödel algebras (also known as Gödel/Dummett algebras, L-algebras, or pre-linear Heyting algebras), viz. the Heyting algebras that satisfy the pre-linearity equation $(a \rightarrow b) \vee(b \rightarrow a) \approx T$. They are the equivalent algebraic semantics of the Gödel/Dummett extension of IPC obtained by adding the linearity axiom $(x \rightarrow y) \vee(y \rightarrow x)$ (see [15, 13] and also [20] for further historical references). In general, if a quasivariety $\mathcal{A}$ is the equivalent algebraic semantics for a sentential logic $\mathcal{L}_{\mathcal{A}}$, then there are connections between algebraic properties in $\mathcal{A}$ and logical properties of $\mathcal{L}_{\mathcal{A}}$. For example, different amalgamation properties in $\mathcal{A}$ correspond to different interpolation properties in $\mathcal{L}_{\mathcal{A}}$ (see for example [5]). This leads us to study amalgamation in Gödel algebras, as reported below.

Duality theory for Heyting algebras is intimately connected with Kripke semantics for IPC. Paralleling these relational semantics there is the topological duality we shall refer to as Priestley/Esakia duality [14]. This specialises Priestley duality for the category $\mathcal{D}$ of bounded distributive lattices to Heyting algebras and provides a primary tool for the study of such algebras. It is well known that a Heyting algebra belongs to $\mathcal{G}_{n}$ if and only if the order of its associated Esakia space is a forest of depth at most $n-2$. Further details are given in Section 4. An overarching

2010 Mathematics Subject Classification. Primary: 06D50; Secondary: 08C20, 06D20 03G25.

Key words and phrases. Gödel algebra, Heyting algebra, natural duality, Esakia duality, amalgamation, coproduct .

The first author was supported by a Marie Curie Intra European Fellowship within the 7th European Community Framework Program (ref. 299401-FP7-PEOPLE-2011-IEF). . 
objective of this paper may be seen as the development of a closer tie-up than hitherto available between natural dualities for the varieties $\mathcal{G}_{n}$ and the Priestley/Esakia duality.

We have aimed to make our results accessible to those interested in Gödel algebras per se and in the applications to amalgamation and coproducts we give in Section 6. Nonetheless, we cannot make our account fully self-contained and shall refer to Clark and Davey's text [4] for background on the fundamentals of natural duality theory. The following summary of certain key events is directed at those already conversant with this theory. It will enable us to set in context for such readers what we achieve in this paper.

It was established in [7] that each variety $\mathcal{G}_{n}$ is endodualisable (so that the alter ego $\left(C_{n} ;\right.$ End $\mathbf{C}_{n}, \mathfrak{T}$ ) yields a duality); in particular $\mathcal{G}_{n}$ is dually equivalent to a category of Boolean spaces acted on by a monoid of continuous maps. While endodualisability of $\mathcal{G}_{n}$ ensures that the alter ego is of an amenable type, the endomorphism monoid End $\mathbf{C}_{n}$ grows exponentially as $n$ increases. This issue was addressed by Davey and Talukder [9, Section 2], with consideration of optimality within the realm of dualities for $\mathcal{G}_{n}$ based on endomorphisms. But there is another approach worthy of consideration. It was to obtain more tractable dualities than those supplied by the NU Duality Theorem, as it applies in particular to distributive lattice-based algebras, that Davey and Werner [10] devised their 'piggyback method'. This leads to a reasonably economical choice of alter ego, which in the case of $\mathcal{G}_{n}$ contains (the graphs of) the members of a family containing both endomorphisms and partial endomorphisms. While natural duality theory was set up from the outset to encompass alter egos containing partial operations, the perception has always been that total structures are to be preferred, whenever possible. However, with a recent spurt of progress in understanding alter egos, the role of partial operations is steadily becoming less mysterious. Moreover, adding partial operations to upgrade a duality to a strong, and hence full, duality is a standard technique for creating full dualities for varieties of lattice-based algebras. Hence including certain partial endomorphisms in an alter ego for a variety $\mathcal{G}_{n}$ may be desirable on grounds of economy, and also the best option for achieving fullness when a full duality is wanted.

The piggyback strategy leans heavily on Priestley duality for $\mathcal{D}$ and it is natural to go one step further and to seek to relate the piggyback natural duality for a $\mathcal{D}$-based quasivariety $\mathcal{A}$ to Priestley duality applied to $\mathrm{U}(\mathbf{A})$, where $\mathrm{U}: \mathcal{A} \rightarrow \mathcal{D}$ is the obvious forgetful functor. Indeed, the germ of the idea for the piggyback method is already present in Davey's proof of his duality for $\mathcal{G}_{n}$ [7, Theorem 2.4]. Esakia, in his review MR0412063 (54 \#192) of [7], observed that it would be interesting to compare Davey's duality for $\mathcal{G}_{n}$ with the duality in Esakia's own 1974 paper [14]. Davey had already taken the first step here: within the proof of [7, Theorem 2.4] he shows how to pass from the natural dual of an algebra $\mathbf{A} \in \mathcal{G}_{n}$ to the Priestley/Esakia dual of its $\mathcal{D}$-reduct. He does this by identifying the latter with a Priestley space obtained as the quotient of the natural dual space of $\mathbf{A}$, where the equivalence relation and the ordering on the quotient are determined by the action of the endomorphisms of $\mathbf{C}_{n}$. The present authors in [3] discussed an analogous process in the context of a piggyback duality for any finitely generated quasivariety of $\mathcal{D}$-based algebras. This translation was developed to facilitate an analysis of coproducts in finitely generated $\mathcal{D}$-based quasivarieties. Here we take these ideas further. For the dualities we present for each class $\mathcal{G}_{n}$, we are able to set up a simple two-way translation, in a functorial way, between the dual categories involved. Simplicity stems from our choices of alter ego; these result a quotienting process which is particularly easy to visualise. But what is much more significant for applications is the bi-directional nature of our translation. We shall refer to the dualities for which such a translation is available as interactive. With an interactive duality to hand we have, in a strong sense, a 'best of both worlds' scenario: we can harness both the categorical virtues of a natural duality and the merits of Priestley duality, specifically its pictorial character and the fact that its is a strong natural duality.

We now outline the structure of this paper and indicate, in somewhat more detail than above, what we achieve. In Section 2 we conduct a detailed analysis of the elements in the hom-sets $\mathcal{G}_{n}\left(\mathbf{A}, \mathbf{C}_{n}\right)$ for $\mathbf{A} \in \mathcal{G}_{n}$. This includes investigation of the images of these maps in $\mathcal{D}(\mathbf{U}(\mathbf{A}), \mathbf{2})$ under $\Phi_{\omega}: x \mapsto \omega \circ x$, where $\omega: \mathrm{U}\left(\mathbf{C}_{n}\right) \rightarrow\{0,1\}$ is the $\mathcal{D}$-homomorphism which sends the top element of $\mathbf{C}_{n}$ to 1 and all other elements to 0 . Some of our results are well known (surjectivity of $\Phi_{\omega}$, for example, is a key component in the validation of Davey and Werner's piggyback duality) 
and certain ingredients are common to our treatment of endomorphisms and that of Davey [7], but other results are new. In particular Lemmas 2.1-2.3 go beyond what appears in the existing literature, and suggest that particular partial endomorphisms and endomorphisms of $\mathbf{C}_{n}$ may be good candidates for inclusion in an alter ego for $\mathbf{C}_{n}$ tailored to a smooth translation between the associated natural duality for $\boldsymbol{\mathcal { S }}_{n}$ and Priestley duality. In Section 3 we combine duality theory's Test Algebra Lemma with an adaptation of the proof of the traditional Piggyback Duality Theorem to obtain a family of new dualities for $\mathcal{G}_{n}$ (for $n \geqslant 4$ ). Each includes in the alter ego $n-3$ partial endomorphisms and one of $n-2$ endomorphisms. In Section 4 we confirm that our new dualities are indeed tailor-made for two-way translation. We adapt the strategy used in [3, Theorem 2.4] to pass, with the aid of $\omega$, from the natural dual of an algebra to the Priestley dual of its reduct. More significantly, the results in Section 2 allow us also to go back again (Theorem 4.6).

We demonstrate the power of our interactive dualities first in Section 5. We are able to show that for each $n$ only one of our $2^{n-3} \times(n-2)$ dualities is full. (We recall that Davey [7] showed that End $\mathbf{C}_{n}$ does not yield a full duality on $\mathcal{G}_{n}$ when $n \geqslant 4$ and it is known that the entire monoid $\operatorname{End}_{\mathrm{p}} \mathbf{C}_{n}$ of partial and total endomorphisms of $\mathbf{C}_{n}$ yields a full duality.) Section 6 is devoted to two different applications: to amalgamation and to coproducts. It follows from results of Maksimova [18] that $\mathcal{G}_{n}$ fails to satisfy the amalgamation property if $n \geqslant 4$, so that not every $V$-formation admits amalgamation in $\boldsymbol{G}_{n}$. We use our two-way translation to determine which $V$-formations do admit amalgamation in $\boldsymbol{G}_{n}$. This result is based on the categorical properties of natural dualities and the fact that Priestley duality maps injective homomorphisms to surjective continuous maps. Finally we extend our work on coproducts [3] by adding Gödel algebras to the catalogue of examples provided there. We employ our two-way translation to give a procedure for describing coproducts in $\mathcal{G}_{n}$ and, in certain cases, in $\mathcal{G}$ too. Our method provides a simple alternative to the procedure presented by D'Antona and Marra [6] in the case of finite Gödel algebras (they employ solely Priestley/Esakia duality) and by Davey [7, Section 5] for algebras in $\boldsymbol{G}_{n}$ (he uses only his natural duality for $\boldsymbol{G}_{n}$ ).

We elect to formulate our principal results about dualities for $\boldsymbol{G}_{n}$ under the assumption $n \geqslant 4$ since to encompass $n=2,3$ would complicate the statements and contribute little that is new. However, mutatis mutandis, the special cases can be fitted into our general scheme, and we make brief comments as we proceed to confirm this.

\section{GÖDEL ALGEBRAS}

An algebra $\mathbf{A}=(A ; \wedge, \vee, \rightarrow, \perp, \top)$ is a Heyting algebra if $(A ; \wedge, \vee, \perp, \top)$ is a bounded distributive lattice and $a \wedge b \leqslant c$ if and only if $a \leqslant b \rightarrow c$. A basic reference for the algebraic properties of Heyting algebras is [1, Chapter IX]. We shall denote the variety of Heyting algebras by $\mathcal{H}$. We make this, and likewise any other class of algebras with which we work, into a category by taking as morphisms all homomorphisms.

It will be important that any Heyting algebra has a reduct in the variety $\mathcal{D}$ of bounded distributive lattices. More precisely, we have a forgetful functor $\mathrm{U}: \mathcal{H} \rightarrow \mathcal{D}$ that on objects sends $\mathbf{A}=(A ; \wedge, \vee, \rightarrow, \perp, \top)$ to $(A ; \wedge, \vee, \perp, \top)$ and sends any morphism, regarded as a map, to the same map. Heyting algebras are rather special amongst algebras with reducts in $\mathcal{D}$ in that the implication $\rightarrow$ is uniquely determined by the underlying order.

The variety $\mathcal{G}$ of Gödel algebras is the subvariety of $\mathcal{H}$ consisting of those algebras which satisfy the pre-linearity equation $(a \rightarrow b) \vee(b \rightarrow a) \approx T$. It is a consequence of pre-linearity that every subdirectly irreducible Gödel algebra is a chain. Moreover $\mathcal{G}$ is generated as a variety by any infinite Heyting chain, and its proper subvarieties are precisely the varieties generated by finite chains [16].

Consider the $n$-element chain with elements $0,1, \ldots, n-1$ labelled so that $0<1<\cdots<n-1$. Then we define $\mathbf{C}_{n}=(\{0,1, \ldots, n-1\} ; \wedge, \vee, \rightarrow, \perp, \top)$, where the constants $\perp$ and $\top$ are taken to be the bounds 0 and $n-1$ and

$$
a \rightarrow b= \begin{cases}\top & \text { if } a \leqslant b \\ b & \text { if } b<a\end{cases}
$$


Trivially, every homomorphic image of $\mathbf{C}_{n}$ is a chain and therefore isomorphic to a subalgebra of $\mathbf{C}_{n}$, whence it follows that $\operatorname{ISP}\left(\mathbf{C}_{n}\right)=\mathbb{H} \mathbb{S P}\left(\mathbf{C}_{n}\right)$. Thus $\mathcal{G}_{n}$, defined earlier to be the quasivariety generated by $\mathbf{C}_{n}$, is also the variety generated by $\mathbf{C}_{n}$. The lattice of subvarieties of the variety $\mathcal{G}$ is the chain

$$
\mathcal{G}_{1} \subseteq \mathcal{G}_{2} \subseteq \cdots \subseteq \mathcal{G} .
$$

Here $\mathcal{G}_{1}$ is generated by the trivial algebra and $\mathcal{G}_{2}$ is term-equivalent to the variety of Boolean algebras.

In [17] it is proved that a Heyting algebra $\mathbf{A}$ is a Gödel algebra if and only if the set of its prime lattice filters forms a forest, that is, the set of prime filters that contain a given prime filter forms a chain (see [16]). Henceforth, following the natural duality approach, we work with homomorphisms into 2 rather than with prime filters: $\mathbf{A}$ is a Gödel algebra if and only if $\mathcal{D}(\mathrm{U}(\mathbf{A}), \mathbf{2})$, ordered pointwise, is a forest. As we have noted already, $\mathbf{A}$ belongs to $\boldsymbol{G}_{n}$ if and only if the forest $\mathcal{D}(\mathrm{U}(\mathbf{A}), 2)$ has depth at most $n-2$. This result is well known but hard to attribute; it can be seen as a consequence of Lemmas 2.1 and 2.2 below. For completeness we belatedly recall the definition of depth. Assume we have a poset $P$ with the property that for every $p \in P$ the up-set $\uparrow p$ does not contain an infinite ascending chain. Then for $p \in P$ we define

$$
d(p)=\max \{|C|-1 \mid C \subseteq \uparrow p \text { and } C \text { is a chain }\} .
$$

If $\{d(p) \mid p \in P\}$ is bounded above, then the depth of $P$ is defined to be $\sup \{d(p) \mid p \in P\}$. We note for future use the fact that in a poset of finite depth the order relation determines and is determined by the associated covering relation, which we denote by $\prec$.

An algebra $\mathbf{A}$ in a quasivariety $\mathcal{A}=\mathbb{I S P}(\mathbf{M})$ is determined by the homomorphisms from $\mathbf{A}$ into M. This fact underlies the centrality in natural duality theory of the hom-sets $\mathcal{A}(\mathbf{A}, \mathbf{M})$ for $\mathbf{A} \in \mathcal{A}$. Accordingly we shall assemble a number of results about homomorphisms from an algebra $\mathbf{A} \in \mathcal{G}_{n}$ into $\mathbf{C}_{n}$. We indicated already in Section 1 the importance of the $\mathcal{D}$-homomorphism $\omega: \mathbf{U}\left(\mathbf{C}_{n}\right) \rightarrow \mathbf{2}$ defined by $\omega(\top)=1$ and $\omega(k)=0$ for $k<\top$. This reflects the key role played by the pre-image of the constant $T$ in the study of homomorphisms between Heyting algebras. For any $\mathbf{A} \in \boldsymbol{G}_{n}$ and any $f \in \mathcal{G}_{n}\left(\mathbf{A}, \mathbf{C}_{n}\right)$ the map $\omega \circ f \in \mathcal{D}(\mathbf{U}(\mathbf{A}), \mathbf{2})$ and $f^{-1}(\top)=(\omega \circ f)^{-1}(1)$.

Lemma 2.1. Let $\mathbf{A} \in \boldsymbol{G}_{n}$ and $x \in \mathcal{G}_{n}\left(\mathbf{A}, \mathbf{C}_{n}\right)$. For each $i \in \operatorname{ran} x \backslash\{0\}$ let $u_{i}: \mathbf{A} \rightarrow \mathbf{2}$ be defined by $u_{i}(a)=1$ if and only if $x(a) \geqslant i$. Then the assignment $i \mapsto u_{i}$ determines a bijection between $\operatorname{ran} x \backslash\{0\}$ and the subset $\uparrow(\omega \circ x)$ of $\mathcal{D}(\mathrm{U}(\mathbf{A}), 2)$. In particular $|\uparrow(\omega \circ x)| \leqslant n-1$ for each $x \in \boldsymbol{G}_{n}\left(\mathbf{A}, \mathbf{C}_{n}\right)$.

Moreover, if $x, y \in \mathbf{G}_{n}\left(\mathbf{A}, \mathbf{C}_{n}\right)$, then

$$
\omega \circ x \prec \omega \circ y \Longleftrightarrow x^{-1}(\top) \subseteq y^{-1}(\top) \text { and }|\operatorname{ran} x|-1=|\operatorname{ran} y| \text {. }
$$

Proof. Certainly, for each $i \in \operatorname{ran} x \backslash\{0\}$, the map $u_{i}$ is a $\mathcal{D}$-homomorphism for which $u_{i} \geqslant \omega \circ x$. To see that the map $i \mapsto u_{i}$ is injective, we argue as follows. Let $i, j \in \operatorname{ran} x \backslash\{0\}$ and assume that $i<j$. Then there exists $a \in \mathbf{A}$ for which $x(a)=j$. This implies that $u_{j}(a)=1$ and $u_{i}(a)=0$, so $u_{i} \neq u_{j}$.

Now let $u \in \uparrow(\omega \circ x)$. Let $k=\min \{x(a) \mid a \in \mathbf{A}$ and $u(a)=1\}$. Note that $k \neq 0$. We claim that $u=u_{k}$. Certainly $u \leqslant u_{k}$. Now assume that $b \in \mathbf{A}$ is such that $u_{k}(b)=1$, that is, $x(b) \geqslant k$. Choose $a \in \mathbf{A}$ such that $u(a)=1$ and $x(a)=k$. Then $u(a \rightarrow b) \geqslant(w \circ x)(a \rightarrow b)=\omega(x(a) \rightarrow$ $x(b))=\omega(\top)=1$. It follows that $u(b) \geqslant u(a \wedge b)=u(a \wedge(a \rightarrow b))=u(a) \wedge u(a \rightarrow b)=1$. Therefore $u_{k} \leqslant u$.

The final claim in the following lemma appears in [10, Section 3.5] (see also the proof of [7, Theorem 2.4]), but the lemma gives additional information. We shall denote the power set of $\{0, \ldots, n-1\}$ by $\wp_{n}$.

Lemma 2.2. Let $\mathbf{A} \in \mathcal{G}_{n}$. Let

$$
T=\left\{(u, V) \in \mathcal{D}(\mathrm{U}(\mathbf{A}), \mathbf{2}) \times \wp_{n} \mid 0, n-1 \in V \text { and }|\uparrow u|+1=|V|\right\} .
$$

Then there exist well-defined and mutually inverse maps

$$
\iota_{\mathbf{A}}: \mathcal{G}_{n}\left(\mathbf{A}, \mathbf{C}_{n}\right) \rightarrow T \quad \text { and } \quad \gamma_{\mathbf{A}}: T \rightarrow \mathcal{G}_{n}\left(\mathbf{A}, \mathbf{C}_{n}\right) .
$$


The first of these is defined by $\iota_{\mathbf{A}}: x \mapsto(\omega \circ x, \operatorname{ran} x)$, for $x \in \mathcal{G}_{n}\left(\mathbf{A}, \mathbf{C}_{n}\right)$. The map $\gamma_{\mathbf{A}}$ is defined in the following way: let $V \in \wp_{n}$ be such that $V=\left\{i_{0}, i_{1}, \ldots i_{m}\right\}$, where $i_{0}=0, i_{m}=n-1$ and $i_{j}<i_{j+1}$ in $\mathbf{C}_{n}$ for $0 \leqslant j \leqslant m$. Then, for $a \in \mathbf{A}$,

$$
\gamma_{\mathbf{A}}(u, V)(a)=i_{k}, \text { where } k=|\{v \in \uparrow u \mid v(a)=1\}| .
$$

In particular, the map $x \mapsto \omega \circ x$ is a surjection from $\mathcal{G}_{n}\left(\mathbf{A}, \mathbf{C}_{n}\right)$ to $\mathcal{D}(\mathrm{U}(\mathbf{A}), \mathbf{2})$.

Proof. Lemma 2.1 tells us that $\iota_{\mathbf{A}}$ is a map from $\mathcal{G}_{n}\left(\mathbf{A}, \mathbf{C}_{n}\right)$ into $T$. Since $|\uparrow u|+1=|V|$, the map $\gamma_{\mathbf{A}}(u, V)$ is well defined for each $(u, V) \in T$. It is straightforward to check that $\gamma_{\mathbf{A}}(u, V)$ is a homomorphism from $\mathbf{A}$ to $\mathbf{C}_{n}$ for each $(u, V) \in T$ and that $\gamma_{\mathbf{A}}$ and $\iota_{\mathbf{A}}$ are mutually inverse.

Combining the fact that the map $x \mapsto \omega \circ x$ is a surjection from $\mathcal{G}_{n}\left(\mathbf{A}, \mathbf{C}_{n}\right)$ to $\mathcal{D}(\mathrm{U}(\mathbf{A}), \mathbf{2})$ with Lemma 2.1, we obtain an alternative proof of the well-known fact that $|\uparrow u| \leqslant n-1$ for each $\mathbf{A} \in \mathcal{G}_{n}$ and $u \in \mathcal{D}(\mathrm{U}(\mathbf{A}), \mathbf{2})$. As a consequence of Lemma 2.2, we can also describe the endomorphisms of $\mathbf{C}_{n}$; cf. [9, Lemma 2.2]. In particular, $e \mapsto$ ran $e$ sets up a bijection from End $\mathbf{C}_{n}$ to $\left\{V \in \wp_{n} \mid 0, n-1 \in V\right\}$.

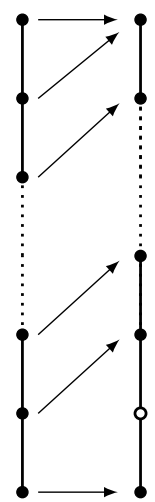

$h_{1}$

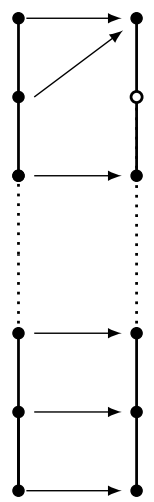

$h_{n-2}$

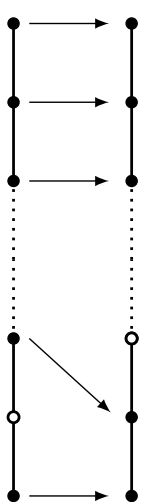

$g_{1}$

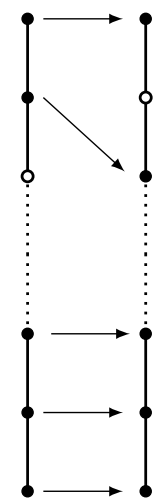

$g_{n-3}$

FIgURE 1. Endomorphisms and partial endomorphisms of $\mathbf{C}_{n}$

We shall make use in the next sections of certain endomorphisms and partial endomorphisms of $\mathbf{C}_{n}$. For $1 \leqslant i \leqslant n-2$ we let $h_{i}$ be the unique endomorphism of $\mathbf{C}_{n}$ with $\operatorname{ran} h_{i}=C_{n} \backslash\{i\}$. More precisely, $h_{i}(x)=x+1$ if $i \leqslant x<n-1$ and $h_{i}(x)=x$ otherwise. (These endomorphisms also appear in [4, Section 2], with $h_{i}$ denoted $e_{i}$, but the use we make of them is different.) For $1 \leqslant i \leqslant n-3$ we define the partial endomorphism $g_{i}$ with domain $C_{n} \backslash\{i\}$ as follows:

$$
g_{i}(x)= \begin{cases}x & \text { if } x \neq i+1 \\ i & \text { if } x=i+1\end{cases}
$$

These maps are indeed partial endomorphisms, none of which extends to an endomorphism. For $1 \leqslant i \leqslant n-3$, let $f_{i}: C_{n} \backslash\{i+1\} \rightarrow C_{n} \backslash\{i\}$ be the inverse of $g_{i}$. For $1 \leqslant i \leqslant n-4$ the map $f_{i}$ is a non-extendable partial endomorphism; $f_{n-3}$ extends to $h_{n-3}$. Figure 1 depicts $h_{1}, h_{n-2}$, $g_{1}$ and $g_{n-3}$; corresponding diagrams of $f_{1}$ and $f_{n-3}$ are obtained from those of $g_{1}$ and $g_{n-3}$ by left-to-right reflection. We fix for future use the following notation: for $n \geqslant 4$,

$$
\Sigma_{n}=\left\{f_{1}, g_{1}\right\} \times \cdots \times\left\{f_{n-3}, g_{n-3}\right\} \times\left\{h_{1}, \ldots, h_{n-2}\right\} .
$$

In Lemma 2.1 we described, for any given $\mathbf{A} \in \mathcal{G}_{n}$, the covering relation on the distinct elements of the set $\left\{\omega \circ x \mid x \in \mathcal{G}_{n}\left(\mathbf{A}, \mathbf{C}_{n}\right)\right\}$. Below we complement this result by demonstrating when elements $\omega \circ x$ and $\omega \circ y$ coincide.

Lemma 2.3. Fix $n \geqslant 4$ and $\sigma \in \Sigma_{n}$. Let $\mathbf{A} \in \mathcal{G}_{n}$ and let $x, y \in \mathcal{G}_{n}\left(\mathbf{A}, \mathbf{C}_{n}\right)$ be such that $x \neq y$. Then the following statements are equivalent: 
(1) $x^{-1}(\top)=y^{-1}(\top)$;

(2) $\omega \circ x=\omega \circ y$;

(3) there exists a finite sequence $z_{0}=x, z_{1}, \ldots, z_{N}=y$ of elements of $\boldsymbol{G}_{n}\left(\mathbf{A}, \mathbf{C}_{n}\right)$ with the property that, for each $0 \leqslant j<N$, there is some $i_{j} \in\{1, \ldots, n-3\}$ such that $z_{j+1}=\sigma_{i_{j}} \circ z_{j}$ or $z_{j}=\sigma_{i_{j}} \circ z_{j+1}$.

Proof. Conditions (1) and (2) are equivalent since $\omega(k)=\top$ if and only if $k=\top$.

Since $f_{i}$ and $g_{i}$ are inverses of each other, without loss of generality we may assume that $\sigma_{i}=g_{i}$ for each $i \leqslant n-3$. Since $g_{i}(k)=\top$ if and only if $k=\top,(3)$ implies (1). It remains to show that (2) implies (3). By Lemma 2.1 and condition (2), $|\operatorname{ran} x|=|\operatorname{ran} y|$. Let $m=|\operatorname{ran} x|=|\operatorname{ran} y|$ and $V=\{0, \ldots, m-2\} \cup\{n-1\}$. Now let $u=\gamma_{\mathbf{A}}(\omega \circ x, V)$ as defined in Lemma 2.2. It is easy to see that either $x=u$ or there exists a sequence of $j_{1}, \ldots, j_{\ell}$ of elements of $\{1, \ldots, n-3\}$ such that $u=g_{j_{1}} \circ g_{j_{2}} \circ \cdots \circ g_{j_{\ell}} \circ x$. Similarly, $y=u$ or $u=g_{k_{1}} \circ g_{k_{2}} \circ \cdots \circ g_{k_{m}} \circ y$ for some $k_{1}, \ldots, k_{m} \in\{1, \ldots, n-3\}$. Since $x \neq y$ we cannot have both $x=u$ and $y=u$. Considering the three remaining possibilities in turn it is easy to see that (3) holds in each case.

\section{Natural DUAlities FOR $\boldsymbol{G}_{n}$}

In what follows we shall use Priestley duality as an ancillary tool. We shall assume familiarity with basic facts concerning this prototypical natural duality (to be found in [4] and [12, Chapter 11]), but we do need to establish notation. We denote the category of Priestley spaces by $\mathcal{P}$. We can express $\mathcal{D}$ and $\mathcal{P}$ as, respectively, $\mathbb{I S P}(\mathbf{2})$ and $\mathbb{I S}_{\mathrm{c}} \mathbb{P}^{+}(\underset{\sim}{\mathbf{2}})$, where $\mathbf{2}=(\{0,1\} ; \wedge, \vee, 0,1)$ and $\mathbf{2}=(\{0,1\} ; \leqslant, \mathcal{T})$; here $\mathcal{T}$ is the discrete topology and $\mathbb{I}_{\mathrm{c}} \mathbb{P}^{+}(\underset{\mathbf{2}}{\sim})$ is the class of isomorphic copies of closed substructures of powers of $\underset{\sim}{\mathbf{2}}$. Here we shall use non-generic symbols $\mathrm{H}$ and $\mathrm{K}$ for the hom-functors $\mathrm{H}=\mathcal{D}(-, \mathbf{2})$ and $\mathrm{K}=\widetilde{\mathcal{P}}(-, \underset{\sim}{\mathbf{2}})$ which set up a dual equivalence between $\mathcal{D}$ and $\mathcal{P}$. Given $\mathbf{L} \in \mathcal{D}$, the evaluation map $k_{\mathbf{L}}: \mathbf{L} \rightarrow \mathbf{K H}(\mathbf{L})$ is defined by $k_{\mathbf{L}}(a)(u)=u(a)$, for $a \in \mathbf{L}$ and $u \in \mathrm{H}(\mathbf{L})$; this map is an isomorphism. We refer to the Priestley space $\mathbf{H}(\mathbf{L})$ as the Priestley dual of $\mathbf{L}$.

We now turn to natural dualities more generally. We shall confine attention to the varieties $\mathcal{G}_{n}=\mathbb{I S P}\left(\mathbf{C}_{n}\right)$ that interest us, referring to [4] any reader who requires an account in a more general setting. We note at the outset that it will suffice for our purposes to consider a more restricted form of alter ego than is allowed for in [4]. (We also remark that we have no need in this paper to consider natural dualities which are multisorted.) We consider a topological structure $\underset{\mathbf{C}_{n}}{\sim}=\left(C_{n} ; G, H, \mathcal{T}\right)$, where $G \subseteq$ End $\mathbf{C}_{n}, H \subseteq \operatorname{End}_{\mathrm{p}} \mathbf{C}_{n} \backslash$ End $\mathbf{C}_{n}$ (the (non-total) partial endomorphisms of $\mathbf{C}_{n}$ ), and $\mathcal{T}$ is the discrete topology. We refer to $\mathbf{C}_{n}$ as an alter ego for $\mathbf{C}_{n}$. We define $\boldsymbol{X}_{n}$ to be the topological quasivariety generated by $\mathbf{C}_{n}$, viz. $\widetilde{X}_{n}=\mathbb{I} \mathbb{S}_{\mathrm{c}} \mathbb{P}^{+}\left(\mathbf{C}_{n}\right)$ : a topological structure of the same type as $\mathbf{C}_{n}$ belongs to $\boldsymbol{X}_{n}$ if and only if it is isomorphic to a closed substructure of a power of $\mathbf{C}_{n}$; here operations and partial operations are lifted pointwise. The superscript ${ }^{+}$serves to indicate that the empty structure is included in $\boldsymbol{X}_{n}$. The morphisms of $\boldsymbol{X}_{n}$ are the continuous structure-preserving maps.

We define hom-functors D: $\mathcal{G}_{n} \rightarrow \boldsymbol{X}_{n}$ and $\mathrm{E}: \boldsymbol{X}_{n} \rightarrow \mathcal{G}_{n}$ as follows:

$$
\begin{array}{ll}
\mathrm{D}: \mathcal{G}_{n} \rightarrow \boldsymbol{X}_{n}, & \left\{\begin{array}{l}
\mathrm{D}(\mathbf{A})=\mathcal{G}_{n}\left(\mathbf{A}, \mathbf{C}_{n}\right) \\
\mathrm{D}(f)=-\circ f,
\end{array}\right. \\
\mathrm{E}: \boldsymbol{X}_{n} \rightarrow \mathcal{G}_{n}, & \left\{\begin{array}{l}
\mathrm{E}(\mathbf{X})=\boldsymbol{X}_{n}\left(\mathbf{X}, \mathbf{C}_{n}\right) \\
\mathrm{E}(\varphi)=-\circ \varphi ;
\end{array}\right.
\end{array}
$$

here $\boldsymbol{G}_{n}\left(\mathbf{A}, \mathbf{C}_{n}\right)$ is considered as a substructure of $\left(\mathbf{C}_{n}\right)^{A}$ and $\boldsymbol{X}_{n}\left(\mathbf{X}, \mathbf{C}_{n}\right)$ inherits its algebra structure pointwise from $\mathbf{C}_{n}$. A crucially important fact is that these functors are well defined. This is a consequence of our assumption that we include in $\mathbf{C}_{n}$ only operations and partial operations which are algebraic. Moreover, for each $\mathbf{A} \in \boldsymbol{G}_{n}$, the evaluation map $e_{\mathbf{A}}$, given by $e_{\mathbf{A}}(a)(x)=x(a)$ (for $a \in \mathbf{A}$ and $x \in \mathrm{D}(\mathbf{A})$ ), is an embedding from $\mathbf{A}$ to $\mathrm{ED}(\mathbf{A})$. Likewise, for each $\mathbf{X} \in \boldsymbol{X}_{n}$, the map $\varepsilon_{\mathbf{X}}$ given by $\varepsilon_{\mathbf{X}}(\varphi)(\alpha)=\alpha(\varphi)$ (for $\varphi \in \mathbf{X}$ and $\alpha \in \mathbf{E}(\mathbf{X})$ ) is an embedding. In categorical terms, $(\mathrm{D}, \mathrm{E}, e, \varepsilon)$ is a dual adjunction between $\boldsymbol{\mathcal { S }}_{n}$ and $\boldsymbol{X}_{n}$ with the unit and counit of the adjunction given by the evaluation maps. (See [4, Chapter 2] for a justification of these assertions 
in a general setting.) Let $\mathbf{A} \in \mathcal{G}_{n}$. We say that $\mathbf{C}_{n}$ (or just $G \cup H$ ) yields a duality on $\mathbf{A}$ if $e_{\mathbf{A}}$ is an isomorphism from $\mathbf{A}$ to $\operatorname{ED}(\mathbf{A})$ and that $G \cup \mathcal{\cup}$ yields a duality on $\boldsymbol{G}_{n}$ if it yields a duality on each $\mathbf{A} \in \mathcal{G}_{n}$. For later use, we say that a dualising alter ego $\mathbf{C}_{n}$ yields a full duality on $\mathcal{G}_{n}$ if $\mathrm{DE}(\mathbf{X}) \cong \mathbf{X}$ for all $\mathbf{X} \in \boldsymbol{X}_{n}$.

We shall need the following result. It is obtained by specialising the Test Algebra Lemma to the very particular situation that concerns us. See [4, Section 8.1] for the general version of this result and contextual discussion. We reiterate that $\mathcal{G}_{n}$ is endodualisable so that the assumptions of Lemma 3.1 are met when $G=$ End $\mathbf{C}_{n}$.

Lemma 3.1. (Test Algebra Lemma, special case) Let $\left(C_{n} ; G, H, \mathcal{T}\right)$ be an alter ego of $\mathbf{C}_{n}$ which is such that $G \subseteq$ End $\mathbf{C}_{n}, H \subseteq \operatorname{End}_{p} \mathbf{C}_{n} \backslash$ End $\mathbf{C}_{n}$ and $G \cup H$ yields a duality on $\boldsymbol{G}_{n}$. Let $e \in G$. Then $(G \backslash\{e\}) \cup H$ yields a duality on $\mathcal{G}_{n}$ provided it yields a duality on the single algebra $\mathbf{C}_{n}$.

Proof. The Test Algebra Lemma in its general form tells us that we can discard $e$ from $G$ without destroying the duality so long as $(G \backslash\{e\}) \cup H$ yields a duality on graph $e$, regarded as an algebra in $\mathcal{G}_{n}$. But the graph of any endomorphism is isomorphic to $\mathbf{C}_{n}$.

We contrast the use of $\mathbf{C}_{n}$ as a test algebra with that employed in [9, proof of Theorem 2.4]. There Davey and Talukder identify a particular generating set $G$ for End $\mathbf{C}_{n}$. They then show that the duality it yields is optimal by showing that $G \backslash\{e\}$ does not yield a duality on the particular algebra $\mathbf{C}_{n}$, for any $e \in G$. This means that they are using the Test Algebra Lemma to guide the choice of an algebra that witnesses indispensability of each member of their set $G$. We use the Test Algebra Lemma in the opposite direction: the lemma tells us that to prove that a given endomorphism $e$ can be dropped from a dualising alter ego it suffices to test this on the single algebra graph $e$-we do not have to verify that $(G \backslash\{e\}) \cup H$ yields a duality on every $\mathbf{A} \in \mathcal{G}_{n}$.

Henceforth, unless indicated otherwise, we shall consider varieties $\mathcal{G}_{n}$ for which $n \geqslant 4$. We include the endomorphism $h_{1}$ (as defined in Section 2) in our alter ego for $\mathbf{C}_{n}$, rather than any alternative endomorphism, because this makes it particularly easy to establish Claim 4 of the proof of Proposition 3.2. We adopt a more even-handed attitude to endomorphisms in Theorem 3.3. The proof of the proposition draws very heavily on the ideas used to prove the Piggyback Duality Theorem [4, Theorem 7.2.1], as this applies to a quasivariety $\operatorname{ISP}(\mathbf{M})$, where $\mathbf{M}$ is a finite algebra with a reduct in $\mathcal{D}$.

Proposition 3.2. Let the partial endomorphisms $g_{1}, \ldots, g_{n-3}$ and endomorphism $h_{1}$ be defined as in Section 2. Then $\left\{g_{1}, \ldots g_{n-3}, h_{1}\right\}$ yields a duality on the algebra $\mathbf{C}_{n}$.

Proof. Observe that the evaluation map $e_{\mathbf{C}_{n}}: \mathbf{C}_{n} \rightarrow \mathrm{ED}\left(\mathbf{C}_{n}\right)$ is injective, and the evaluation map $k_{\mathrm{U}\left(\mathbf{C}_{n}\right)}: \mathrm{U}\left(\mathbf{C}_{n}\right) \rightarrow \operatorname{KHU}\left(\mathbf{C}_{n}\right)$ is an isomorphism, and so surjective. We want to show that $e_{\mathbf{C}_{n}}$ is surjective. Now we bring in the critical, but entirely elementary, observation that it will suffice to construct an injective map $\Delta: \operatorname{ED}\left(\mathbf{C}_{n}\right) \rightarrow \operatorname{KHU}\left(\mathbf{C}_{n}\right)$ (see [4, proof of Piggyback Duality Theorem 7.2.1] or [10]).

Recall that $\omega: \mathrm{U}\left(\mathbf{C}_{n}\right) \rightarrow \mathbf{2}$ denotes the $\mathcal{D}$-morphism with $\omega^{-1}(1)=\{n-1\}$ and that for each $u \in \mathrm{HU}\left(\mathbf{C}_{n}\right)$ we can find $x \in \mathrm{D}\left(\mathbf{C}_{n}\right)$ such that $u=\omega \circ x$. We may now attempt to define $\Delta$ as follows. Given $\varphi \in \operatorname{ED}\left(\mathbf{C}_{n}\right)$ let

$$
(\Delta(\varphi))(u)=(\Delta(\varphi))(\omega \circ x)=\omega(\varphi(x)) .
$$

We now establish a series of claims. These combine with the observations above to prove the proposition.

1. $\Delta$ is a well-defined map.

We have already observed that every element of $\operatorname{HU}\left(\mathbf{C}_{n}\right)$ is of the form $\omega \circ x$ for some $x \in \mathrm{D}\left(\mathbf{C}_{n}\right)$. We must now check that, for $x$ and $y$ in $\mathrm{D}\left(\mathbf{C}_{n}\right)=$ End $\mathbf{C}_{n}$ and $\varphi \in \operatorname{ED}\left(\mathbf{C}_{n}\right)$,

$$
\omega \circ x=\omega \circ y \Longrightarrow \omega(\varphi(x))=\omega(\varphi(y)) \text {. }
$$

Suppose first that $y=g_{i} \circ x$ for some $g_{i}$. Then $\varphi(x)=\top$ if and only if $\varphi(y)=g_{i}(\varphi(x))=\top$. We argue likewise when $x=g_{i} \circ y$. Hence, by Lemma 2.3, $\varphi(x)=\top$ if and only if $\varphi(y)=\top$. Since $\omega(j)=1$ if and only if $j=\top$, our claim is proved. 
2. $\Delta(\varphi)$ is order-preserving for each $\varphi \in \operatorname{ED}\left(\mathbf{C}_{n}\right)$.

For $1 \leqslant i \leqslant n-1$, let $u_{i}: \mathbf{C}_{n} \rightarrow \mathbf{2}$ be the map determined by $u_{i}^{-1}(1)=\uparrow i$. It is trivial to check that the set $\left\{u_{1}, \ldots, u_{n-1}\right\}$ coincides with $\operatorname{HU}\left(\mathbf{C}_{n}\right)$ and $u_{1}>u_{2}>\cdots>u_{n-1}$.

Let $1 \leqslant i<j<n-1$, so $u_{j}<u_{i}$. Assume that $(\Delta(\varphi))\left(u_{j}\right)=1$. We wish to show that $(\Delta(\varphi))\left(u_{i}\right)=1$. For each $k$ such that $i \leqslant k \leqslant j$, let $x_{k}=\gamma_{\mathbf{A}}\left(u_{k},(\{0\} \cup\{n-k \ldots, n-1\})\right)$, where the map $\gamma_{\mathbf{A}}$ is as defined in Lemma 2.2. It follows that $\omega \circ x_{i}=u_{i}$ and $\omega \circ x_{j}=u_{j}$. Then $1=(\Delta(\varphi))\left(u_{j}\right)=\omega\left(\varphi\left(x_{j}\right)\right)$, that is, $\varphi\left(x_{j}\right)=\top$. Clearly $x_{k}=h_{1} \circ x_{x+1}$ whenever $i \leqslant k<j$. Since $\varphi\left(x_{j}\right)=\top, h_{1}(\top)=\top$, and $\varphi$ preserves $h_{1}$, it follows that $\varphi\left(x_{i}\right)=\top$. Therefore $(\Delta(\varphi))\left(u_{i}\right)=\omega\left(\varphi\left(x_{i}\right)\right)=1$.

3. For each $\varphi \in \operatorname{ED}\left(\mathbf{C}_{n}\right)$ the map $\Delta(\varphi): \operatorname{HU}\left(\mathbf{C}_{n}\right) \rightarrow \mathbf{2}$ is continuous.

This is immediate because $\mathbf{C}_{n}$ is finite.

4. $\Delta$ is injective.

Suppose that $\varphi, \psi \in \mathrm{ED}\left(\mathbf{C}_{n}\right)$ with $\varphi \neq \psi$. Pick $x \in \mathrm{D}\left(\mathbf{C}_{n}\right)$ such that $\varphi(x) \neq \psi(x)$ in $\mathbf{C}_{n}$. Without loss of generality, assume that $\varphi(x)<\psi(x)$. Let $j=(n-1)-\psi(x)$. Then $h_{1}^{j}(\psi(x))=\top$ and $h_{1}^{j}(\varphi(x)) \neq \top$ (where $h_{1}^{j}$ denotes the $j$-fold composition of $h_{1}$ if $j>0$ and the identity map if $j=0)$. Since $\varphi$ and $\psi$ preserve $h_{1}$,

$$
(\Delta(\psi))\left(\omega \circ h_{1}^{j} \circ x\right)=\omega\left(\psi\left(h_{1}^{j} \circ x\right)\right)=\omega\left(h_{1}^{j}(\psi(x))\right)=\omega\left(h_{1}^{j}(\psi(x))\right)=1
$$

and

$$
(\Delta(\varphi))\left(\omega \circ h_{1}^{j} \circ x\right)=\omega\left(\varphi\left(h_{1}^{j} \circ x\right)\right)=\omega\left(h_{1}^{j}(\varphi(x))\right)=\omega\left(h_{1}^{j}(\varphi(x))\right)=0 .
$$

Therefore $\Delta(\varphi) \neq \Delta(\psi)$.

The following theorem supplies a family of alter egos each of which dualises $\boldsymbol{G}_{n}$. In Section 5, we shall see that, even if the natural dualities presented in Theorem 3.3 are closely connected, they have significantly different properties. We recall that the definition of $\Sigma_{n}$ was given in Section 2 .

Theorem 3.3. Let $\sigma \in \Sigma_{n}$. Then $\mathbf{C}_{n}^{\sigma}=\left(C_{n} ; \sigma, \mathcal{T}\right)$ yields a duality on $\boldsymbol{G}_{n}=\mathbb{I} \mathbb{S P}\left(\mathbf{C}_{n}\right)$.

Proof. We first note that Lemma 3.1 and Proposition 3.2 combine to tell us that the alter ego $\left(C_{n} ; g_{1}, \ldots, g_{n-3}, h_{1}, \mathcal{T}\right)$ yields a duality on $\mathcal{G}_{n}$.

For any $i$, the maps $g_{i}$ and $f_{i}$ are interchangeable because their graphs are mutual converses. We note that $h_{1}=f_{1} \circ \cdots \circ f_{i-1} \circ h_{i}$ for $2 \leqslant i \leqslant n-2$. Hence (see [4, Section 2.4]) $h_{1}$ is entailed by $f_{1}, \ldots, f_{n-3}$ and $h_{i}$. Therefore $\mathbf{C}_{n}^{\sigma}$ yields a duality for any choice of $\sigma$ from $\Sigma_{n}$.

We remark that we could use the Test Algebra Lemma to prove that each of the dualities presented in Theorem 3.3 is optimal; cf. [9, Theorem 2.4]. The technique is standard and we do not include details here.

We briefly consider $\mathcal{G}_{3}$. Here End $\mathbf{C}_{3}=\left\{\operatorname{id}_{\mathbf{C}_{3}}, h_{1}\right\}$, and there are no non-extendable endomorphisms to consider. We could define $\Sigma_{3}=\left\{h_{1}\right\}$ and so bring $n=3$ within the scope of Theorem 3.3. But this adds nothing that is new: already in [7] the alter ego $\left(C_{3} ; h_{1}, \mathcal{T}\right)$ was shown to yield a duality on $\mathcal{G}_{3}$. For $n=2$ there is even less that is worth saying, since $\operatorname{End}\left(\mathbf{C}_{2}\right)=\left\{\operatorname{id}_{C_{2}}\right\}$ and there are no non-extendable partial endomorphisms. The duality for $\mathcal{G}_{2}$ associated with $\Sigma_{2}$, defined to be $\emptyset$, is just Stone duality for Boolean algebras.

\section{From natural duality to Priestley/Esakia duality And Back again}

The main objective in this section is to investigate how the dualities presented in Theorem 3.3 facilitate translation from the categorically well-behaved natural duality set-up to the more pictorial representation afforded by Priestley/Esakia duality for Heyting algebras. Before demonstrating how the translation operates we briefly recall the Priestley/Esakia duality. This has a long history, and has been rediscovered and reformulated many times. By way of reference we note here Esakia's paper [14] and also the recent paper [2].

The relative pseudocomplement in a Heyting algebra is uniquely determined by the underlying lattice order. More precisely, we may assert that the forgetful functor $U: \mathcal{H} \rightarrow \mathcal{D}$ is faithful and $\mathrm{U}: \mathcal{H} \rightarrow \mathrm{U}(\mathcal{H})$ is part of a categorical equivalence (actually an isomorphism); the inverse 
$\mathrm{V}: \mathrm{U}(\mathcal{H}) \rightarrow \mathcal{H}$ maps each bounded distributive lattice $\mathbf{L}$ that admits a relative pseudocomplement to the unique Heyting algebra $\mathbf{A}$ such that $\mathbf{U}(\mathbf{A})=\mathbf{L}$.

An algebra $\mathbf{L} \in \mathcal{D}$ can be identified with its second dual $\mathbf{K}(\mathbf{X})$, where $\mathbf{X}=\mathbf{H}(\mathbf{L})$. There exists a Heyting algebra $\mathbf{B}$ with $\mathrm{U}(\mathbf{B})=\mathrm{K}(\mathbf{X})$ if and only if the Priestley space $\mathbf{X}=(X ; \leqslant, \mathcal{T})$ has the property that $\downarrow O$ is $\mathcal{T}$-open whenever $O$ is $\mathcal{T}$-open; if this condition is satisfied we call $\mathbf{X}$ an Esakia space. Given Esakia spaces $\mathbf{X}$ and $\mathbf{Y}$, a continuous order-preserving map $\varphi: \mathbf{Y} \rightarrow \mathbf{X}$ is such that $\mathrm{K}(\varphi): \mathrm{K}(\mathbf{X}) \rightarrow \mathrm{K}(\mathbf{Y})$ preserves the relative pseudocomplement if and only $\varphi$ is an Esakia morphism, meaning that $\varphi(\uparrow y)=\uparrow \varphi(y)$ for all $y \in Y$. In summary, there is a dual equivalence between the category of Heyting algebras and the category of Esakia spaces (with Esakia morphisms), obtained by restricting the duality between $\mathcal{D}$ and $\mathcal{P}$ to the subcategory $\mathrm{U}(\mathcal{H})$ and a certain subcategory $\mathcal{E}$ of $\mathcal{P}$.

As observed earlier, a Heyting algebra is a Gödel algebra if and only if the associated Esakia space is a forest. In our formulation of the duality trees grow downwards. Restricting the functors $\mathrm{HU}$ and $\left.\mathrm{VK}\right|_{\mathcal{E}}$ we obtain a dual equivalence between the category $\mathcal{G}$ of Gödel algebras and the category $\mathcal{F}$ of Esakia spaces whose order structure is a forest and Esakia morphisms.

The category $\mathcal{G}_{n}$ is dually equivalent to the full subcategory $\mathcal{F}_{n}$ of Esakia spaces whose objects are forests of depth at most $n-2$. Figure 2 summarises the various dual equivalences relating to Priestley/Esakia duality and their restrictions to full subcategories, shown by unlabelled vertical arrows.

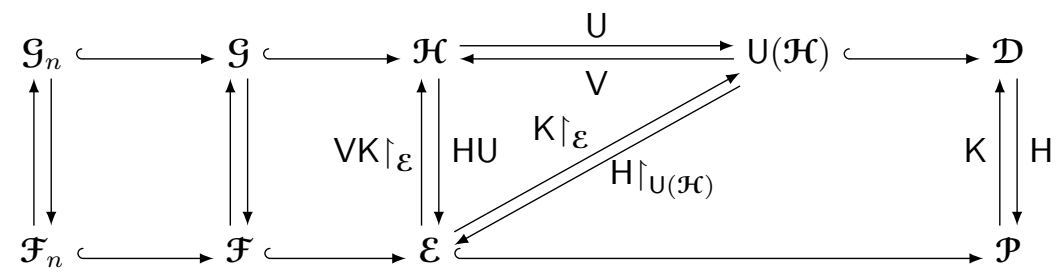

Figure 2. Priestley/Esakia duality for Gödel algebras

For fixed $n \geqslant 4$ and each choice of $\sigma \in \Sigma_{n}$, we shall use $\mathrm{D}^{\sigma}$ and $\mathrm{E}^{\sigma}$ to denote the functors determined by the alter ego $\mathbf{C}_{n}^{\sigma}$ of $\mathbf{C}_{n}$. Our immediate aim is to relate the dual space $\mathrm{D}^{\sigma}(\mathbf{A})$ to the Priestley/Esakia dual HU( $\widetilde{\mathbf{A}})$. Some word of explanation is needed before we demonstrate how to do this. Let $\mathbf{y}^{\sigma}$ denote the full subcategory of $\mathbb{I S}_{\mathrm{c}} \mathbb{P}^{+}\left(\mathbf{C}_{n}^{\sigma}\right)$ whose class of objects is $\mathbb{I}\left(\mathrm{D}^{\sigma}\left(\boldsymbol{G}_{n}\right)\right)$. From Theorem 3.3 and the Priestley/Esakia duality for $\widetilde{\mathcal{G}}_{n}$, it is straightforward to see that $\mathcal{F}_{n}$ and $\mathbf{y}^{\sigma}$ are equivalent categories. Therefore one may ask: why present a description of $\mathrm{D}^{\sigma}(\mathbf{A})$ from $\mathrm{HU}(\mathbf{A})$, and vice versa, if this can be obtained using $\mathbf{A}$ as a stepping stone? The answer is that to prove the trivial fact that $\mathcal{F}_{n}$ and $\boldsymbol{y}^{\sigma}$ are equivalent is not our final goal. We want to reveal the very special connection between these categories which will be our primary tool in the final sections of the paper.

Assume we have any finitely generated (quasi)variety $\mathcal{A}$ of distributive lattice-based algebras with forgetful functor $U: \mathcal{A} \rightarrow \mathcal{D}$. In [3, Section 2] we presented a procedure for passing from the natural dual of an algebra $\mathbf{A} \in \mathcal{A}$ to the Priestley dual $\mathbf{H U}(\mathbf{A})$ when the natural duality under consideration was obtained by the piggyback method. Here we carry out an analogous process, but now based on any of the dualities we established in Theorem 3.3. We shall do this by proving a variant of [3, Theorem 2.4], as this theorem applies to the special case in which $\mathcal{A}=\mathcal{G}_{n}$. This result-Theorem 4.3-achieves more than the direct specialisation of the general result. The reason for this lies in the way in which, for $\mathbf{A} \in \mathcal{G}_{n}$, the layers of the Priestley space $\mathrm{HU}(\mathbf{A})$ are derived from the action of the maps $g_{i}$ (or $f_{i}$ ) on $\mathbf{D}(\mathbf{A})$, and how the lifting of the chosen endomorphism $h_{j}$ relates these layers. (It is convenient to visualise $\mathrm{HU}(\mathbf{A})$ as being comprised of layers, each layer consisting of the elements at a particular depth; see Fig. 3 relating to Example 4.4.)

Before we begin we recap on the form taken by the objects of the dual category $\boldsymbol{X}_{n}^{\sigma}=\mathbb{I} \mathbb{S}_{\mathrm{c}} \mathbb{P}^{+}\left(\mathbf{C}_{n}^{\sigma}\right)$, where $\sigma \in \Sigma_{n}$. These are topological structures $\mathbf{X}=\left(X ; \sigma_{1}^{\mathbf{x}}, \sigma_{2}^{\mathbf{x}}, \ldots, \sigma_{n-2}^{\mathbf{x}}, \mathcal{T}^{\mathbf{x}}\right)$, where the partial 
operations $\sigma_{i}^{\mathbf{x}}$ (for $1 \leqslant i \leqslant n-3$ ) and the operation $\sigma_{n-2}^{\mathbf{x}}$ are obtained by pointwise lifting of the corresponding operations $\sigma_{i}$ on $\mathbf{C}_{n}$ and the domain of each partial operation is a closed substructure of $\mathbf{X}$ (see [4, Chapter 2] for details). Let $\simeq_{\mathbf{X}}^{\sigma}$ be the binary relation defined on $X$ by $x \simeq_{\mathbf{X}}^{\sigma} y$ if and only if either $x=y$ or there exists a sequence $z_{0}=x, \ldots, z_{N}=y \in \mathbf{X}$ such that, for each $j \in\{0, \ldots, N-1\}$, there exists $i_{j} \in\{1, \ldots, n-3\}$ such that $z_{j+1}=\sigma_{i_{j}}^{\mathbf{x}} \circ z_{j}$ or $z_{j}=\sigma_{i_{j}}^{\mathbf{x}} \circ z_{j+1}$. Then $\simeq_{\mathrm{x}}^{\sigma}$ is an equivalence relation on $X$. The definition of $\simeq_{\mathrm{x}}^{\sigma}$ is motivated by Lemma 2.3 , which can be recast as follows.

Lemma 4.1. Let $\sigma \in \Sigma_{n}$. Let $\mathbf{A} \in \mathcal{G}_{n}$ and $\mathbf{X}=\mathrm{D}^{\sigma}(\mathbf{A})$ and $x, y \in \mathbf{X}$. Then

$$
x^{-1}(\top)=y^{-1}(\top) \Longleftrightarrow \omega \circ x=\omega \circ y \Longleftrightarrow x \simeq_{\mathbf{x}}^{\sigma} y
$$

The cluttered notation adopted below is temporarily necessary because we shall work simultaneously with more than one alter ego. We denote the equivalence class of $x \in X$ under $\simeq_{\mathrm{x}}^{\sigma}$ by $[x]_{\simeq_{\mathrm{X}}^{\sigma}}$. We now define a relation $\prec_{\mathrm{X}}^{\sigma}$ on $X / \simeq_{\mathbf{x}}^{\sigma}$ as follows:

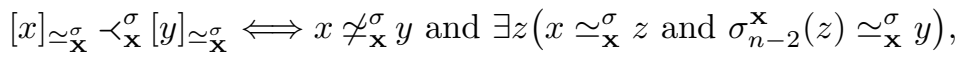

and let $\leqslant_{\mathbf{x}}^{\sigma}$ be the reflexive, transitive closure of $\prec_{\mathbf{x}}^{\sigma}$. Taking the reflexive, transitive closure of the antisymmetric relation $\prec_{\mathbf{x}}^{\sigma}$ does not destroy antisymmetry, so $\leqslant_{\mathrm{x}}^{\sigma}$ is a partial order.

Lemma 4.2. Let $\sigma, \tau \in \Sigma_{n}$. Let $\mathbf{A}$ be an algebra in $\mathbf{G}_{n}$ and let $\mathbf{X}=\mathrm{D}^{\sigma}(\mathbf{A})$ and $\mathbf{X}^{\prime}=\mathrm{D}^{\tau}(\mathbf{A})$ be the associated dual spaces. Then

(i) $\simeq_{\mathbf{x}}^{\sigma}$ and $\simeq_{\mathbf{x}^{\prime}}^{\tau}$ are equal;

(ii) $\leqslant_{\mathbf{x}}^{\sigma}$ and $\leqslant_{\mathbf{x}^{\prime}}^{\tau}$ are equal.

Moreover, for any $\sigma \in \Sigma_{n}$, the relation $\leqslant_{\mathrm{x}}^{\sigma}$ is a partial order on $X / \simeq_{\mathrm{x}}^{\sigma}$ of depth at most $n-2$, for which $\prec_{\mathbf{x}}^{\sigma}$ is the associated covering relation.

Proof. (i) follows directly from Lemma 4.1. Since we now know that the equivalence relations on $X=\mathcal{G}_{n}\left(\mathbf{A}, \mathbf{C}_{n}\right)$ obtained from $\sigma$ and $\tau$ are the same we shall write simply $\simeq$ for the relation and

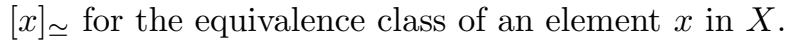

We now prove (ii). Let $x, y \in X$ be such that $[x]_{\simeq} \prec_{\mathrm{x}}^{\sigma}[y]_{\simeq \text {. Then }} x \not 4 y$ and there exists $z \in[x]_{\simeq}$ for which $\sigma_{n-2}^{\mathrm{X}}(z) \in[y]_{\simeq}$. Since $h_{j}(n-2)=n-1$ for any $j \in\{1, \ldots, n-2\}$, it follows that, for $a \in \mathbf{A}$,

$$
\left(\omega \circ \sigma_{n-2}^{\mathbf{x}}(z)\right)(a)=1 \Longleftrightarrow z(a) \in\{n-2, n-1\} \Longleftrightarrow\left(\omega \circ \tau_{n-2}^{\mathbf{x}}(z)\right)(a)=1 .
$$

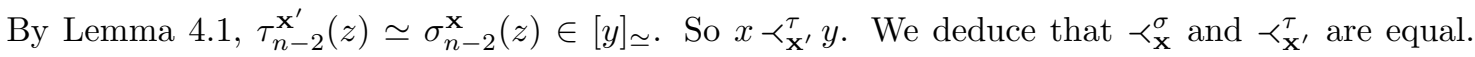
Therefore $\leqslant_{\mathrm{x}}^{\sigma}$ coincides with $\leqslant_{\mathrm{x}^{\prime}}^{\tau}$.

The final assertions follow from Lemma 2.1 and the way in which the order on $X / \simeq$ is defined.

Theorem 4.3. Let $\sigma \in \Sigma_{n}$. Let $\mathbf{A} \in \mathcal{G}_{n}$ and $\mathbf{X}=\mathrm{D}^{\sigma}(\mathbf{A})$ be its dual space. Let $Y=X / \simeq_{\mathbf{x}}^{\sigma}$ and let $\mathcal{T}$ be the quotient topology derived from the topology of $\mathrm{D}^{\sigma}(\mathbf{A})$. Then $\mathbf{Y}=(Y ; \leqslant \sigma, \mathcal{T})$ is a Priestley space isomorphic to $\mathrm{HU}(\mathbf{A})$.

Proof. By Lemma 4.2 we may assume that $\sigma=\left(g_{1}, \ldots, g_{n-3}, h_{1}\right)$. We shall write $\simeq$ in place of $\simeq_{\mathbf{x}}^{\sigma}$ and omit subscripts from equivalence classes and from the order and covering relations on $X / \simeq$.

We know that the map $\Phi_{\omega}: x \mapsto \omega \circ x$ from $\mathrm{D}(\mathbf{A})$ to $\mathrm{HU}(\mathbf{A})=\left(Z ; \leqslant_{\mathbf{z}}, \mathcal{T}^{\mathbf{z}}\right)$ is surjective. Arguing just as in the proof of $\left[3\right.$, Theorem 2.3] we proved that $\left(Z ; \mathcal{T}^{\mathbf{z}}\right)$ is homeomorphic to the quotient space $\left(X / \operatorname{ker}\left(\Phi_{\omega}\right) ; \mathcal{T}^{\mathbf{x}} / \operatorname{ker}\left(\Phi_{\omega}\right)\right)$. From the definition of $\Phi_{\omega}$, we have $\Phi_{\omega}(x)=\Phi_{\omega}(x)$ if and only if $\omega \circ x=\omega \circ y$. By Lemma 4.1, $\operatorname{ker}\left(\Phi_{\omega}\right)$ coincides with the relation $\simeq$ described in terms of the liftings of $g_{1}, \ldots, g_{n-3}$. So we have identified $(\mathrm{D}(\mathbf{A}) / \simeq ; \mathcal{T})$ with $\left(Z ; \mathcal{T}^{\mathbf{z}}\right)$.

It remains to reconcile the order of the quotient space with that of $\operatorname{HU}(\mathbf{A})$. Since we are working with posets of finite depth it suffices to consider the covering relations. First suppose that $[x] \prec[y]$. So there exists $z$ such that $x \simeq z, y \simeq h_{1}^{\mathbf{x}}(z)$. Since $i \leqslant h_{1}(i)$ for each $i \in \mathbf{C}_{n}$, we have, for $a \in \mathbf{A}$,

$$
\left.\omega(x(a))=\omega(z(a)) \leqslant \omega\left(h_{1}(z(a))\right)=\left(\omega \circ h_{1}^{\mathbf{x}}(z)\right)(a)\right)=\omega(y(a))
$$

and hence $\omega \circ x \leqslant \omega \circ y$ in $\operatorname{HU}(\mathbf{A})$. 
Conversely, assume that $\omega \circ y$ covers $\omega \circ x$ in $\mathrm{HU}(\mathbf{A})$. Assume $d(\omega \circ y)=j$ and $d(\omega \circ x)=j+1$. By Lemma 2.2, there exists $z \in \mathcal{G}_{n}\left(\mathbf{A}, \mathbf{C}_{n}\right)$ such that $\omega \circ z=\omega \circ x$ and $\operatorname{ran}(z)=\{0\} \cup \uparrow(n-1-j)$. By Lemma 4.1, $z \simeq x$. Now observe that $\operatorname{ran}\left(h_{1}^{\mathbf{x}} \circ z\right)=\{0\} \cup \uparrow(n-j)$ and so $\omega \circ x=\omega \circ z<\omega \circ\left(h_{1}^{\mathbf{x}}(z)\right)$. Since $\uparrow(\omega \circ x)$ is a chain and $d\left(\omega \circ\left(h_{1}^{\mathbf{x}}(z)\right)\right)=j=d(\omega \circ y)$, we have $\omega \circ\left(h_{1}^{\mathbf{x}}(z)\right)=\omega \circ x$. Therefore $h_{1}^{\mathbf{x}}(z) \simeq y$. Hence $[x] \leqslant[y]$.

A retrospective look at [7] is due here. There are clear similarities between our proof of Theorem 4.3 and Davey's original proof of endodualisability of $\mathcal{G}_{n}[7$, Theorem 2.4]. Lemma 4.1 establishes that, for each $\mathbf{A} \in \mathcal{G}_{n}$, our relation $\simeq_{\mathrm{D}(\mathbf{A})}^{\sigma}$ coincides with the relation $R$ defined in the proof of [7, Theorem 2.4]. But there is an important point to note. The relations $\simeq$ and $\prec$ are defined using the lifting of the (partial and non-partial) operations $\sigma_{i}$. Therefore, they are available in every space in $\boldsymbol{X}=\mathbb{I} \mathbb{S}_{c} \mathbb{P}^{+}\left(\mathbf{C}_{n}^{\sigma}\right)$, and not only those of the form $\mathrm{D}(\mathbf{A})$ for some $\mathbf{A} \in \mathcal{G}_{n}$. This difference becomes crucial in the following sections when we determine which dualities are full and which $V$-formations admit amalgamation.

We now take a break from theory to discuss how translation works in practice.

Example 4.4. Fix $n=5$ and $\sigma=\left(g_{1}, g_{2}, h_{3}\right)$. We illustrate the passage from the natural dual to the Priestley/Esakia dual for the algebra $\mathbf{C}_{5}$. Here the elements of $\mathbf{X}=\mathrm{D}^{\sigma}\left(\mathbf{C}_{5}\right)$ are exactly the endomorphisms of $\mathbf{C}_{5}$, on which $g_{1}, g_{2}$ and $h_{3}$ act by composition. We label each endomorphism $e$ of $\mathbf{C}_{5}$ by writing ran $e \backslash\{0,4\}$ (which uniquely determines $e$ ) as a string, as indicated in Fig. 3 . For endomorphisms $e$ and $f$ we have $(e, f) \in \operatorname{graph} g_{i}^{\mathbf{x}}$ if and only if

$$
\forall a \in \mathbf{C}_{5}\left((e(a), f(a)) \in \operatorname{graph} g_{i}\right)
$$

for $i=1,2$. In order for this to hold it is necessary that $i \notin \operatorname{dom} e$ and $i+1 \notin \operatorname{ran} f$. In the figure, the solid arrows arrows indicate the action of $h_{3}$. Dashed and dotted arrows indicate, respectively, the action of $g_{1}$ and of $g_{2}$.

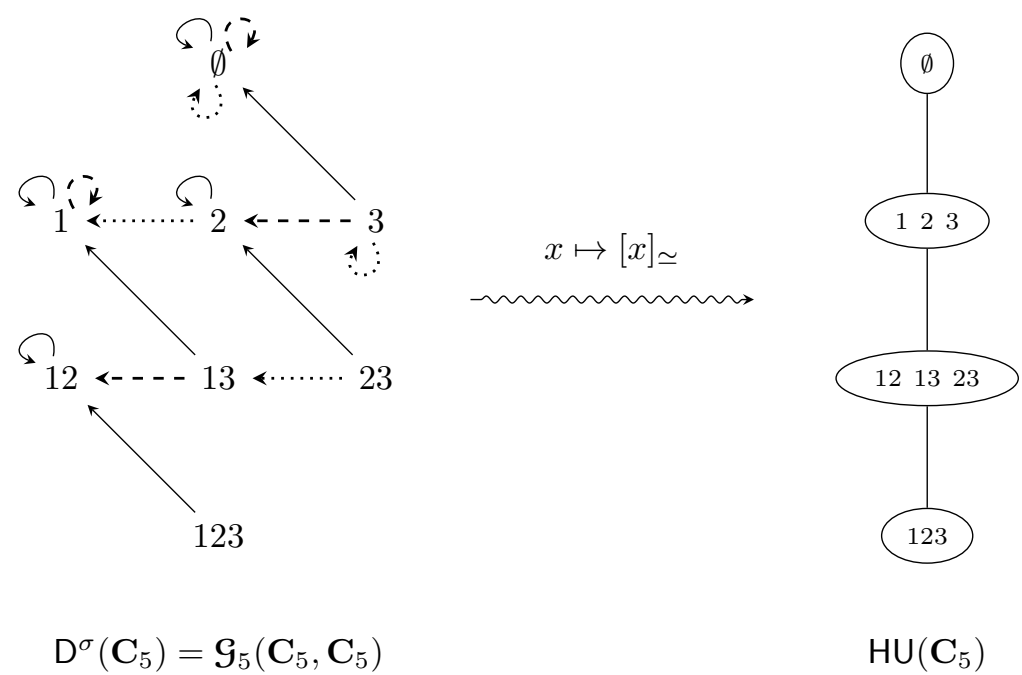

Figure 3. Translation applied to the algebra $\mathbf{C}_{5}$ in $\mathcal{G}_{5}$

Of course we have a special situation here because $\mathbf{C}_{5}$ is a chain. In general each layer of the dual space will not be a single $\simeq$-equivalence class.

We elected here to use the endomorphism $h_{3}$ in the alter ego, rather than the alternatives $h_{1}$ and $h_{2}$ supplied by Theorem 3.3, because the action of $h_{3}$ on End $\mathbf{C}_{5}$ is especially simple. However one feature of the translation is present whichever of $h_{1}, h_{2}$ and $h_{3}$ we include the alter ego: $\simeq$ is determined solely by $g_{1}$ and $g_{2}$, whereas the ordering amongst $\simeq$-equivalence classes is determined solely by $h_{i}$, whichever value of $i$ we choose. 
We should draw attention, however, to Theorem 5.1 below in which we show that in any application in which we need a full (or equivalently a strong) duality for $\boldsymbol{G}_{n}$, then we must use $h_{1}$ rather than any other $h_{i}$.

We now seek to demonstrate that the process for passing from $\mathrm{D}\left(\mathbf{C}_{n}\right)$ to $\operatorname{HU}\left(\mathbf{C}_{n}\right)$ (for $\left.n \geqslant 4\right)$ is much less transparent using a duality based solely on endomorphisms (as in [7, 9]) than when we use any of the variants supplied by Theorem 3.3.

Example 4.5. We shall consider the alter ego $\left(C_{5} ; h_{1}, h_{2}, h_{3}, \mathcal{T}\right)$ for $\mathcal{G}_{5}$. As shown by Davey and Talukder [9, Theorem 2.4], this yields an optimal duality. In Fig. 4, the action of $h_{1}, h_{2}$ and $h_{3}$ on End $\mathbf{C}_{n}$ is shown by dashed, dotted and solid arrows, respectively. It can be seen that these maps do encode $\simeq$ and that, on the associated quotient, we can recover the ordering of $\operatorname{HU}\left(\mathbf{C}_{5}\right)$. What does emerge clearly from this example is that translation from an endomorphism-based duality to Priestley/Esakia duality can be quite complicated, even on very simple objects. Moreover fully reconciling our approach with that in [7] is not a trivial exercise in practice, though the theory ensures that it is, of course, possible.

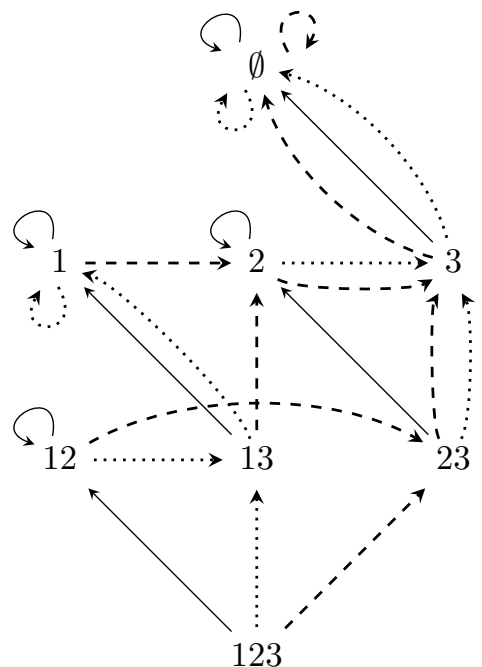

Figure 4. The dual of $\mathbf{C}_{5}$ for the duality for $\mathcal{G}_{5}$ with alter ego $\left(C_{5} ; h_{1}, h_{2}, h_{3}, \mathcal{T}\right)$

Theorem 4.6 presents the other half of the two-way translation process between $\mathcal{F}_{n}$ and $\mathbf{y}^{\sigma}$, for a given $\sigma \in \Sigma_{n}$, as it applies to an object $\operatorname{HU}(\mathbf{A})$, where $\mathbf{A} \in \boldsymbol{G}_{n}$. Lemma 2.2 sets up, for the given algebra $\mathbf{A}$, mutually inverse bijections $\iota_{\mathbf{A}}$ and $\gamma_{\mathbf{A}}$ between $\mathcal{G}_{n}\left(\mathbf{A}, \mathbf{C}_{n}\right)$ and a specified subset of $\mathrm{HU}(\mathbf{A}) \times \wp_{n}$. Starting from the Esakia space $\mathrm{HU}(\mathbf{A})$, we form suitable pairs with elements of $\wp_{n}$ and $\mathrm{HU}(\mathbf{A})$. We then form a topological structure $\mathbf{X}$ by equipping our set of pairs with a topology and operations $\sigma_{i}^{\mathbf{X}}$ and establish that $\mathbf{X}$ is isomorphic to the natural dual space $\mathbf{D}^{\sigma}(\mathbf{A})$. We carry out this construction using only the order and topological structure of $\operatorname{HU}(\mathbf{A})$ (see the remarks following the theorem for the significance of this). The Priestley/Esakia duality applied to $\mathbf{A} \in \mathcal{G}_{n}$ tells us that the evaluation map $k_{\mathbf{A}}: \mathbf{A} \rightarrow \operatorname{KHU}(\mathbf{A})$ is an isomorphism and the sets of the form $k_{\mathbf{A}}(a)$, as a ranges over $\mathbf{A}$, are precisely the clopen up-sets in $\mathrm{HU}(\mathbf{A})$. Moreover, we recall that $\mathrm{D}(\mathbf{A})$ is topologised with the subspace topology induced by the product topology on $\left(C_{n}\right)^{A}$, where the topology on $C_{n}$ is discrete. These observations underlie the way topology is handled in the theorem.

Theorem 4.6. Let $\mathbf{A}$ be an algebra in $\boldsymbol{G}_{n}$ and let $\mathrm{HU}(\mathbf{A})=(Y ; \leqslant, \mathcal{T})$. Let

$$
X=\left\{(y, U) \in Y \times \wp_{n}|| U \mid=d(y)+1 \text { and } 0, n-1 \in U\right\} .
$$


Define partial maps $g_{i}^{\mathbf{x}}$ and $f_{i}^{\mathbf{x}}$ on $X$ as follows, where the domains are given by the indicated restrictions:

$$
\begin{array}{ll}
g_{i}^{\mathbf{x}}(y, U)=\left(y, g_{i}(U)\right) & \text { if } i \notin U, \\
f_{i}^{\mathbf{x}}(y, U)=\left(y, f_{i}(U)\right) & \text { if } i+1 \notin U,
\end{array}
$$

and total maps $h_{j}^{\mathbf{x}}$ given by

$$
h_{j}^{\mathbf{x}}(y, U)= \begin{cases}\left(y, h_{j}(U)\right) & \text { if } n-2 \notin U \\ \left(s(y), h_{j}(U)\right) & \text { otherwise }\end{cases}
$$

here $s$ denotes the function which, on $\mathrm{HU}(\mathbf{A})$ - a forest of finite depth-maps each non-maximal point to its unique upper cover and fixes each maximal point.

For each clopen up-set $W$ of $\mathrm{HU}(\mathbf{A})$ and each $i \in \mathbf{C}_{n}$, let

$$
A_{W, i}=\{(y, U) \in X \mid i \in U \text { and }|\downarrow i \cap U|=|\uparrow x \cap W|+1\} .
$$

Let $\sigma \in \Sigma_{n}$. Then $\mathrm{D}^{\sigma}(\mathbf{A}) \cong \mathbf{X}=\left(X ; \sigma_{1}^{\mathbf{x}}, \sigma_{1}^{\mathbf{x}}, \ldots, \sigma_{n-2}^{\mathbf{x}}, \mathcal{T}^{\mathbf{x}}\right)$, where $\mathcal{T}^{\mathbf{x}}$ is the topology generated by the family of sets of the form $A_{W, i}$.

Proof. Lemma 2.2 sets up mutually inverse bijections $\iota_{\mathbf{A}}: \mathcal{G}_{n}\left(\mathbf{A}, \mathbf{C}_{n}\right) \rightarrow X$ and $\gamma_{\mathbf{A}}: X \rightarrow \mathcal{G}_{n}\left(\mathbf{A}, \mathbf{C}_{n}\right)$, where $\iota_{\mathbf{A}}: x \mapsto(\omega \circ x, \operatorname{ran} x)$. From the description of $\gamma_{\mathbf{A}}$ given there, for each $a \in \mathbf{A}$ and $i \in \mathbf{C}_{n}$, we have $\left(\gamma_{\mathbf{A}}(y, U)\right)(a)=i$ if and only if $i \in U$ and $|\downarrow i \cap U|-1=\left|\left\{u \in \uparrow y \mid u \in k_{\mathbf{A}}(a)\right\}\right|$. Thus

$$
\gamma_{\mathbf{A}}\left(A_{k_{\mathbf{A}}(a), i}\right)=\{x \in \mathrm{D}(\mathbf{A}) \mid x(a)=i\}
$$

and hence $\iota_{\mathbf{A}}$ determines a homeomorphism between $\left(X ; \mathcal{T}^{\mathbf{X}}\right)$ and $\mathrm{D}^{\sigma}(\mathbf{A})$.

In what follows $g_{i}^{\mathrm{D}(\mathbf{A})}, f_{i}^{\mathrm{D}(\mathbf{A})}$ and $h_{j}^{\mathrm{D}(\mathbf{A})}$ denote the lifting of the (partial) maps $g_{i}, f_{i}$ and $h_{j}$ to $\mathcal{G}_{n}\left(\mathbf{A}, \mathbf{C}_{n}\right)$ for each $i \in\{1, \ldots, n-3\}$ and $j \in\{1, \ldots, n-2\}$.

Let $i \in\{1, \ldots, n-3\}$ and $x \in \mathcal{G}_{n}\left(\mathbf{A}, \mathbf{C}_{n}\right)$. Then $x \in \operatorname{dom}\left(g_{i}^{\mathrm{D}(\mathbf{A})}\right)$ if and only if $\operatorname{ran} x \subseteq \operatorname{dom} g_{i}$, that is, if $i \notin \operatorname{ran} x$. In this case

$$
\begin{aligned}
\iota_{\mathbf{A}}\left(g_{i}^{\mathrm{D}(\mathbf{A})}(x)\right) & =\iota_{\mathbf{A}}\left(g_{i} \circ x\right)=\left(\omega \circ g_{i} \circ x, \operatorname{ran}\left(g_{i} \circ x\right)\right) \\
& =\left(\omega \circ f, g_{i}(\operatorname{ran} x)\right)=g_{i}^{\mathbf{x}}\left(\iota_{\mathbf{A}}(x)\right) .
\end{aligned}
$$

The proof that $\iota_{\mathbf{A}} \circ f_{i}^{\mathrm{D}(\mathbf{A})}=f_{i}^{\mathbf{x}} \circ \iota_{\mathbf{A}}$ is similar. Finally, let $j \in\{1, \ldots, n-2\}$ and $x \in \mathcal{G}_{n}\left(\mathbf{A}, \mathbf{C}_{n}\right)$. Then

$$
\iota_{\mathbf{A}}\left(h_{j}^{\mathrm{D}(\mathbf{A})}(x)\right)=\iota_{\mathbf{A}}\left(h_{j} \circ x\right)=\left(\omega \circ h_{j} \circ x, h_{j}(\operatorname{ran} x)\right) .
$$

Here we have two cases. If $n-2 \notin \operatorname{ran} x$ then $\omega \circ h_{j} \circ x=\omega \circ x$. If $n-2 \in \operatorname{ran} x$ then $\omega \circ h_{j} \circ x=s(\omega \circ x)$. We deduce that $\iota_{\mathbf{A}}$ preserves $\sigma_{i}$ for $1 \leqslant i \leqslant n-2$.

A remark is in order here on what we have really achieved in Theorem 4.6. We have already observed that our 'going back' construction builds (up to isomorphism) $\mathrm{D}^{\sigma}(\mathbf{A}) \in \mathbf{y}^{\sigma}$ solely from the topology and order of the Esakia space $\operatorname{HU}(\mathbf{A})$ (as encoded by the map $s$ ). That is, the construction is performed without directly involving the algebra $\mathbf{A}$. This means that we can carry out the procedure on any Esakia space in $\mathcal{F}_{n}$, regardless of whether or not the space is explicitly represented in the form $\mathrm{HU}(\mathbf{A})$ (as it can be, certainly).

We introduced the category $\mathbf{y}^{\sigma}$ earlier but did not then give an explicit description of the equivalence between this category and $\mathcal{F}_{n}$ indicated in Fig. 5 . We can now remedy this omission. Define $\mathrm{F}^{\sigma}: \boldsymbol{y}^{\sigma} \rightarrow \mathcal{F}_{n}$ on objects by letting

$$
\mathbf{F}^{\sigma}(\mathbf{X})=\left(X / \simeq_{\mathbf{X}}^{\sigma} ; \leqslant_{\mathbf{X}}^{\sigma}, \mathcal{T}^{\mathbf{x}} / \simeq_{\mathbf{X}}^{\sigma}\right) .
$$

Now define $\mathrm{F}^{\sigma}(\eta)$, for each $\eta \in \mathbf{y}^{\sigma}(\mathbf{X}, \mathbf{Y})$, as follows:

$$
\mathrm{F}^{\sigma}(\eta)\left([x]_{\simeq_{\mathbf{X}}^{\sigma}}\right)=[\eta(x)]_{\simeq_{\mathbf{Y}}^{\sigma}} \quad(\text { for } x \in \mathbf{X}) .
$$

The fact that $\mathrm{F}^{\sigma}(\eta)$ is well defined follows from the definition of $\simeq_{\mathbf{Y}}^{\sigma}$ and the fact that $\eta$ preserves $\sigma_{1}, \ldots, \sigma_{n-3}$. Since $\eta$ preserves $\sigma_{n-2}$, it is straightforward to check that $\mathrm{F}^{\sigma}(\eta)$ is an Esakia morphism. Then $\mathrm{F}^{\sigma}$ is a functor naturally equivalent to $\mathrm{HU} \uparrow_{\mathcal{G}_{n}} \mathrm{E}^{\sigma}\left\lceil_{\mathbf{y}^{\sigma}}\right.$. 


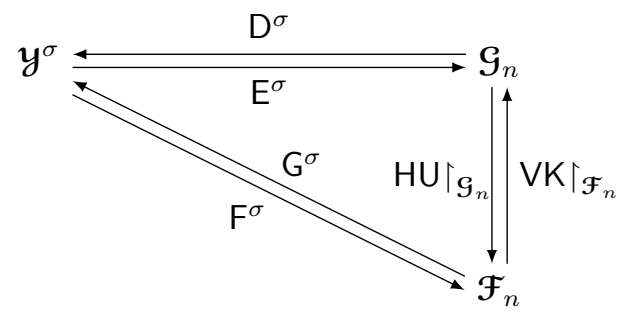

FIgURE 5. The equivalence set up by the functors $\mathrm{F}^{\sigma}$ and $\mathrm{G}^{\sigma}$

As with $\mathrm{F}^{\sigma}$, the assignment $\mathbf{Y} \mapsto \mathrm{G}^{\sigma}(\mathbf{Y})=\left(X ; \sigma_{1}^{\mathbf{x}}, \ldots, \sigma_{n-1}^{\mathbf{x}}, \mathcal{T}^{\mathbf{x}}\right)$ can be extended to a functor from $\mathcal{F}_{n}$ into $\mathbf{y}^{\sigma}$. In this case we will not present explicitly the action of $\mathrm{G}^{\sigma}$ on maps. For our purposes, it is enough to observe that Theorems 4.3 and 4.6 imply that $\mathrm{G}^{\sigma}$ can be made into a functor naturally equivalent to $\left.\mathrm{D}^{\sigma} \mathrm{V} \mathrm{K}\right|_{\mathcal{F}_{n}}$; equivalently, $\mathrm{F}^{\sigma}$ together with $\mathrm{G}^{\sigma}$ determine a categorical equivalence between $\mathbf{y}^{\sigma}$ and $\mathcal{F}_{n}$.

In the same way as we did in Section 3 we end this section with a comment about $\mathcal{G}_{3}$ and $\mathcal{G}_{2}$. Using the alter ego $\mathbf{C}_{3}=\left(\mathbf{C}_{3} ; h_{1}, \mathfrak{T}\right)$ for $\mathbf{C}_{3}$, Davey [7, pp. 126-127] shows how to obtain $\mathrm{H}(\mathbf{A})$ from $\mathrm{D}(\mathbf{A})$ he first observes that $\mathrm{H}(\mathbf{A})$ and $\mathrm{D}(\mathbf{A})$ are homeomorphic as topological spaces. In our terms this means that $x \simeq y$ if and only if $x=y$. This is actually the natural way to define $\simeq$ in the absence of partial endomorphisms. Moreover, if we identify $D(\mathbf{A}) / \simeq$ with $D(\mathbf{A})$ then the order Davey defines on $\mathbf{D}(\mathbf{A})$ is exactly the reflexive (transitive) closure of $\prec$. This is only one side of the translation; the other direction can be obtained by the same construction and argument used in Theorem 4.6. For $\mathcal{G}_{2}$, the translation between the duality yielded by $\left(C_{2} ; \mathcal{T}\right)$ and the Priestley/Esakia duality is essentially that whereby a Boolean space is regarded as a special case of a Priestley space.

\section{The QUEST FOR FUlL DUALITIES}

Our first application of the translations developed in Section 4 is to pick out the full dualities from among the dualities we developed in Theorem 3.3.

In Theorem 5.1 we show that, for $n \geqslant 4$, all choices of $\sigma \in \Sigma_{n}$ except $\sigma=\left(g_{1}, \ldots, g_{n-3}, h_{1}\right)$ lead to dualities which are not full. Our strategy is similar to one used by Davey; see his proof that End $\mathbf{C}_{n}$ fails to dualise $\mathcal{G}_{n}$ fully when $n \geqslant 4$ [7, p. 127].

The primary tool for establishing that a natural duality is full is to establish that it is strong (see [4, Chapter 3] for the definitions and discussion). The dualities for $\mathcal{G}_{2}$ and for $\mathcal{G}_{3}$ yielded by the alter egos $\left(C_{2} ; \mathcal{T}\right)$ and $\left(C_{3} ; h_{1}, \mathcal{T}\right)$ contain no partial operations. They are known to be strong (see [4, Theorem 4.2.3(ii)] for the case $n=3$ ) and hence full. When $n=4$, the fact that $\mathbf{C}_{n}^{\sigma}$, with $\sigma=\left(g_{1}, h_{1}\right)$, determines a (strong and hence) full duality for $\mathcal{G}_{4}$ was proved by Davey and Talukder in [9, Theorem 6.1]. We shall show that, for any $n \geqslant 4$, the dualising set $\left\{g_{1}, \ldots, g_{n-3}, h_{1}\right\}$ yields a full duality. Our proof uses Theorems 4.3 and 4.6. It is this technique, and the fact that fullness is obtained directly, and not via strongness, that we wish to accentuate here. We note also that Davey's proof of fullness of his endomorphism-based duality for $\mathcal{G}_{3}$ [7, pp. 126-127] may be seen as essentially a very special case of our method. Our full dualities are necessarily strong; see [8, pp. 13-14], where Gödel algebras are called relative Stone Heyting algebras. (The paper [8], a stepping stone along the way to the final resolution in the negative of the longstanding "ful equals strong?" question, identifies various well-known varieties for which non-strong full dualities cannot be found.) As an aside, we note that a small generating set for the monoid $\operatorname{End}_{\mathrm{p}} \mathbf{C}_{n}$ is needed if axiomatisation of the dual category is to feasible. We can claim to have set up as good a full duality as is possible for addressing the axiomatisation problem for general $n$. However in this paper we shall not seek an extension of [9, Theorem 6.1], which relies both on a suitable generating set for $\operatorname{End}_{\mathrm{p}} \mathbf{C}_{4}$ being found by hand and on standardness arguments (see [9, Section 3]). 


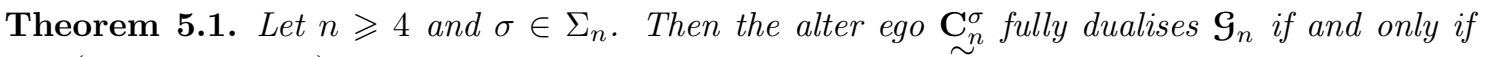
$\sigma=\left(g_{1}, \ldots, g_{n-3}, h_{1}\right)$.

Proof. Assume first that $\sigma \neq\left(g_{1}, \ldots, g_{n-3}, h_{1}\right)$. We divide the problem into two cases. Both proofs employ the same tool. Since the functors $\mathrm{G}^{\sigma}: \mathcal{F}_{n} \rightarrow \mathbf{y}_{n}^{\sigma}$ and $\mathrm{F}^{\sigma}: \mathbf{y}_{n}^{\sigma} \rightarrow \mathcal{F}_{n}$ determine a categorical equivalence, $\mathbf{X}$ is isomorphic to $\mathrm{G}^{\sigma}\left(\mathrm{F}^{\sigma}(\mathbf{X})\right)$ for every $\mathbf{X} \in \mathbf{y}_{n}^{\sigma}$. Therefore we present a space $\mathbf{X}$ in $\mathbb{I S}_{\mathrm{c}} \mathbb{P}^{+}\left(\mathbf{C}_{n}^{\sigma}\right)$ and we observe that $\mathbf{X}$ is not isomorphic to $\mathrm{G}^{\sigma}\left(\mathrm{F}^{\sigma}(\mathbf{X})\right)$, which proves that $\mathbf{y}_{n}^{\sigma} \neq \mathbb{I S}_{\mathrm{c}} \mathbb{P}^{+}\left(\mathbf{C}_{n}^{\sigma}\right)$, that is, $\mathbf{C}_{n}^{\sigma}$ does not yield a full duality. In the proof below we shall omit subscripts and superscripts, for example from $\simeq$, where these are clear from the context.

Case 1: Assume that $\sigma_{i}=f_{i}$ for some $i \in\{1, \ldots, n-3\}$.

Let $j$ be such that $\sigma_{j}=f_{j}$ and $\sigma_{i}=g_{i}$ for any $j<i \leqslant n-3$. Let $\mathbf{X}$ be the subspace of $\mathbf{C}_{n}^{\sigma}$ whose universe $X$ is $\{j+1, \ldots, n-1\}$. Observe that $\operatorname{dom} \sigma_{i}^{\mathbf{x}}=X$ if $i \neq j$ and $\operatorname{dom} \sigma_{j}^{\mathbf{x}}=\{j+2, \ldots, n-1\}$. Now assume $\mathbf{X} \in \mathbf{y}^{\sigma}$. By assumption, the quotient space $X / \simeq$ has two elements $\{j+1, \ldots, n-2\}$ and $\{n-1\}$. Then $\mathbf{F}^{\sigma}(\mathbf{X})=\left(\left\{[n-2]_{\simeq},[n-1]_{\simeq}\right\} ; \leqslant, \mathcal{T}\right)$, where $\mathcal{T}$ is the discrete topology and $[n-2]_{\simeq}<[n-1]_{\simeq}$. The universe of $\mathrm{G}^{\sigma}\left(\mathrm{F}^{\sigma}(\mathbf{X})\right)$ is $\left\{\left([n-1]_{\simeq}, \emptyset\right),([n-2] \simeq,\{1\}), \ldots,\left([n-2]_{\simeq},\{n-2\}\right)\right\}$. It follows that $\left|\mathrm{G}^{\sigma}\left(\mathrm{F}^{\sigma}(\mathbf{X})\right)\right|=n-1$ and $|X|=n-1-j$. Therefore $\mathrm{G}^{\sigma}\left(\mathrm{F}^{\sigma}(\mathbf{X})\right)$ and $\mathbf{X}$ are not isomorphic.

Case 2: Assume that $\sigma_{i}=g_{i}$ for $1 \leqslant i \leqslant n-3$ and $\sigma_{n-2}=h_{i}$, where $i \neq 1$.

With this assumption $h_{i}(1)=1$, and so $\{1, n-1\}$ is a closed subuniverse of $\mathbf{C}_{n}^{\sigma}$. Let $\mathbf{X}$ be the subspace of $\mathbf{C}_{n}^{\sigma}$ whose universe $X$ is $\{1, n-1\}$. Observe that $\operatorname{dom} \sigma_{i}^{\mathbf{x}}=X$ if $i \neq 1$ and $\operatorname{dom} \sigma_{1}^{\mathbf{x}}=\left\{n-1 \tilde{\}}\right.$. Now assume $\mathbf{X} \in \mathbf{y}^{\sigma}$. Since $\sigma_{i}=g_{i}$ for $1 \leqslant i \leqslant n-3$, the definition of $\simeq$ implies that $X / \simeq$ has two classes $\{1\}$ and $\{n-1\}$. Because $h_{i}(1)=1$ and $h_{i}(n-1)=n-1$, the space $\mathrm{F}^{\sigma}(\mathbf{X})$ is $\left(\left\{[1]_{\simeq},[n-1]_{\simeq}\right\} ;=, \mathcal{T}\right)$, where $\mathcal{T}$ is the discrete topology. The universe of $\mathbf{Z}=\mathrm{G}^{\sigma}\left(\mathrm{F}^{\sigma}(\mathbf{X})\right)$ is $\left\{\left([1]_{\simeq}, \emptyset\right),\left([n-1]_{\simeq}, \emptyset\right)\right\}$ and $\operatorname{dom} \sigma_{1}^{\mathbf{Z}}=Z$. Since $\operatorname{dom} \sigma_{1}^{\mathbf{X}}=\{n-1\}$, the spaces $\mathbf{Z}$ and $\mathbf{X}$ are not isomorphic.

Now assume $\sigma=\left(g_{1}, \ldots, g_{n-3}, h_{1}\right)$. Since $\mathbf{C}_{n}^{\sigma}$ determines a duality on $\mathcal{G}_{n}$, for each non-empty set $S$, the space $\left(\mathbf{C}_{n}^{\sigma}\right)^{S}$ is isomorphic to the dual space of the $S$-generated free algebra $\mathbf{F}_{\boldsymbol{G}_{n}}(S)$ in $\mathcal{G}_{n}$ [4, Corollary 2.24]. Therefore, to prove that $\mathbf{C}_{n}^{\sigma}$ determines a full duality, it is enough to prove that each closed substructure $\mathbf{X}$ of $\mathrm{D}^{\sigma}\left(\mathbf{F}_{\mathcal{G}_{n}}(S)\right)$, for some set $S$, is isomorphic to the dual space of some algebra in $\mathcal{G}_{n}$.

Let us fix a non-empty set $S$ and a closed substructure $\mathbf{X}$ of $\left(\mathbf{C}_{n}^{\sigma}\right)^{S}$. Let $x \in \mathbf{X}$ and $y \in\left(\mathbf{C}_{n}^{\sigma}\right)^{S}$ be such that $x \simeq y$. We claim that $y \in \mathbf{X}$. By the definition of $\simeq$ there is no loss of generality in assuming that $x=g_{i}^{\mathbf{x}}(y)$ or $y=g_{i}^{\mathbf{x}}(x)$ for some $i \in\{1, \ldots, n-3\}$. If $y=g_{i}^{\mathbf{x}}(x)$, since $\mathbf{X}$ is a closed substructure of $\left(\mathbf{C}_{n}^{\sigma}\right)^{S}$, it follows directly that $y \in \mathbf{X}$. If $x=g_{i}^{\mathbf{x}}(y)$ then, for each $s \in S$, we have $x_{s}=g_{i}\left(y_{s}\right)$ and hence $x_{s} \in \operatorname{dom} f_{i}$ and $f_{i}\left(x_{s}\right)=y_{s}$. Now observe that for $i \in\{1, \ldots, n-1\}$, the partial endomorphism $f_{i}$ equals $g_{i-1} \circ g_{i-2} \circ \cdots \circ g_{2} \circ g_{1} \circ h_{1} \circ g_{n-3} \circ g_{n-2} \circ \cdots \circ g_{i+2} \circ g_{i+1}$. Then

$$
y=g_{i-1}^{\mathbf{x}} \circ g_{i-2}^{\mathbf{x}} \circ \cdots \circ g_{2}^{\mathbf{x}} \circ g_{1}^{\mathbf{x}} \circ h_{1}^{\mathbf{x}} \circ g_{n-3}^{\mathbf{x}} \circ g_{n-2}^{\mathbf{x}} \circ \cdots \circ g_{i+2}^{\mathbf{x}} \circ g_{i+1}^{\mathbf{x}}(x),
$$

and we have established our claim.

Let $\mathbf{A}=\mathbf{F}_{\mathcal{G}_{n}}(S)$ and let $\mathbf{Y}=\left(\mathrm{D}^{\sigma}(\mathbf{A}) / \simeq ; \leqslant, \mathcal{T}^{\mathbf{Y}}\right)$ be as defined in Theorem 4.3. The fact that, for each $x \in \mathbf{X}$, the class $[x] \simeq$ is contained in $\mathbf{X}$ implies that $\mathbf{X} / \simeq \subseteq \mathrm{D}^{\sigma}(\mathbf{A}) / \simeq$. Let $\mathbf{Z}$ be the substructure of $\mathbf{Y}$ whose universe is $\mathbf{X} / \simeq$. Since $\mathbf{X}$ is a closed subset of $\mathrm{D}^{\sigma}(\mathbf{A})$ and $\mathcal{T}^{\mathbf{Y}}$ is the quotient topology, $\mathbf{X} / \simeq$ is a closed subset of $\left(D^{\sigma}(\mathbf{A}) / \simeq ; \mathcal{T}^{\mathbf{Y}}\right)$. From the fact that $\mathbf{X}$ is closed under $h_{1}$ and the definition of $\leqslant$ in $\mathrm{D}^{\sigma}(\mathbf{A}) / \simeq$, it follows that $\mathbf{X} / \simeq$ is an up-set in $\left(\mathrm{D}^{\sigma}(\mathbf{A}) / \simeq ; \leqslant\right)$. We conclude that $\mathbf{Z}$ belongs to $\mathcal{F}_{n}$. Therefore there exists $\mathbf{B} \in \mathcal{G}_{n}$ such that $\mathrm{HU}(\mathbf{B}) \cong \mathbf{Z}$. By Theorem 4.6, $\mathrm{D}^{\sigma}(\mathbf{B}) \cong \mathrm{G}^{\sigma}(\mathbf{Z})$.

By Theorems 4.3 and 4.6 , the map $\iota_{\mathbf{A}}: \mathrm{D}^{\sigma}(\mathbf{A}) \rightarrow \mathrm{G}^{\sigma} \mathrm{F}^{\sigma}\left(\mathrm{D}^{\sigma}(\mathbf{A})\right)$, defined by $\iota_{\mathbf{A}}(x)=(\omega \circ$ $x, \operatorname{ran} x)$, sets up an isomorphism. Since for $x \in \mathbf{X}$ the class $[x]_{\simeq}$ is contained in $\mathbf{X}$, the restriction of $\iota_{\mathbf{A}}$ to $\mathbf{X}$ determines a bijection between $\mathbf{X}$ and $G^{\sigma}(\mathbf{Z})$. Moreover, $\iota_{\mathbf{A}} \uparrow_{\mathbf{X}}$ is an isomorphism between $\mathbf{X}$ and $G^{\sigma}(\mathbf{Z})$ in the category $\mathbb{I S}_{\mathbf{c}} \mathbb{P}^{+}\left(\mathbf{C}_{n}^{\sigma}\right)$. Putting the components of the proof together we deduce that $\mathbf{X} \cong \mathrm{G}^{\sigma}(\mathbf{Z}) \cong \mathrm{D}^{\sigma}(\mathbf{B})$. 


\section{Applichtions: amalgamation and coproducts}

Our second application of our interactive duality theory concerns amalgamation in varieties of Gödel algebras. Our main result here is Theorem 6.4. Along the way we expose the interrelation between satisfaction of the amalgamation property in a finitely generated quasivariety and the existence of an alter ego which is a total structure yielding a strong duality: the result given in Lemma 6.1 is not new, but we do present a simpler and more self-contained proof of it than that of $[4$, Lemma 5.3].

Given a class of algebras $\mathcal{A}$, a $V$-formation is a quintuple $\left(\mathbf{A}, \mathbf{B}, \mathbf{C}, f_{B}, f_{C}\right)$ where $\mathbf{A}, \mathbf{B}, \mathbf{C} \in \mathcal{A}$, and $f_{B} \in \mathcal{A}(\mathbf{A}, \mathbf{B})$ and $f_{C} \in \mathcal{A}(\mathbf{A}, \mathbf{C})$ are injective homomorphisms. A $V$-formation $\left(\mathbf{A}, \mathbf{B}, \mathbf{C}, f_{B}, f_{C}\right)$ admits amalgamation if there exist an algebra $\mathbf{D} \in \mathcal{A}$ and embeddings $h_{B}: \mathbf{B} \rightarrow \mathbf{D}$ and $h_{C}: \mathbf{C} \rightarrow$ $\mathbf{D}$ such that $h_{B} \circ f_{B}=h_{C} \circ f_{C}$. The class $\mathcal{A}$ has the amalgamation property if every $V$-formation admits amalgamation.

Let $\mathcal{A}$ be a quasivariety. It is well known that $\mathcal{A}$ admits all colimits and in particular pushouts, also known as fibred coproducts (see [19, Chapter III] for definitions and properties of colimit, pushout, and coproduct). Let $\left(\mathbf{A}, \mathbf{B}, \mathbf{C}, f_{B}, f_{C}\right)$ be a $V$-formation in $\mathcal{A}$. Then $\left(\mathbf{A}, \mathbf{B}, \mathbf{C}, f_{B}, f_{C}\right)$ admits amalgamation if and only if the pushout maps $p_{B}: \mathbf{B} \rightarrow \mathbf{B} \coprod_{\mathbf{A}} \mathbf{C}$ and $p_{C}: \mathbf{B} \rightarrow \mathbf{B} \coprod_{\mathbf{A}} \mathbf{C}$ are embeddings. With this in mind, we shall focus below on the properties of pushouts, and particularly on when pushout maps are embeddings.

Lemma 6.1. Let $\mathcal{A}=\mathbb{I S P}(\mathbf{M})$ be the quasivariety generated by a finite algebra $\mathbf{M}$ and assume that there is a total structure $\underset{\sim}{\mathbf{M}}$ which yields a strong duality on $\mathcal{A}$. Then $\mathcal{A}$ has the amalgamation property.

Proof. Let $\mathrm{D}: \mathcal{A} \rightarrow \mathbb{I S}_{\mathrm{c}} \mathbb{P}^{+}(\underset{\sim}{\mathbf{M}})$ and $\mathrm{E}: \mathbb{I S}_{\mathrm{c}} \mathbb{P}^{+}(\underset{\sim}{\mathbf{M}}) \rightarrow \mathcal{A}$ be the functors determined by $\underset{\sim}{\mathbf{M}}$. By $[4$, Theorem 6.1.2 and Exercise 6.1], under our assumptions a homomorphism $h$ in $\mathcal{A}$ is an embedding if and only if $\mathrm{D}(h)$ is surjective.

Let $\left(\mathbf{A}, \mathbf{B}, \mathbf{C}, f_{B}, f_{C}\right)$ be a $V$-formation in $\mathcal{A}$. Then $\mathrm{D}\left(f_{B}\right)$ and $\mathrm{D}\left(f_{C}\right)$ are surjections. Let $\mathbf{X}$ be the fibred product $\mathrm{D}(\mathbf{B}) \stackrel{\mathrm{D}\left(f_{B}\right)}{\longrightarrow} \mathrm{D}(\mathbf{A}) \stackrel{\mathrm{D}\left(f_{C}\right)}{\longleftarrow} \mathrm{D}(\mathbf{C})$. That is, $\mathbf{X}$ is the subspace of $\mathrm{D}(\mathbf{B}) \times \mathrm{D}(\mathbf{C})$ whose universe is

$$
X=\left\{(x, y) \in \mathrm{D}(\mathbf{B}) \times \mathrm{D}(\mathbf{C}) \mid \mathrm{D}\left(f_{B}\right)(x)=\mathrm{D}\left(f_{C}\right)(y)\right\} .
$$

Since $\mathrm{D}\left(f_{B}\right)$ and $\mathrm{D}\left(f_{C}\right)$ are surjective, the projection maps $\pi_{B}: X \rightarrow \mathrm{D}(\mathbf{B})$ and $\pi_{C}: X \rightarrow \mathrm{D}(\mathbf{C})$ are also surjective. Since the duality is full, it follows that $\mathrm{E}(\mathbf{X})$ is the pushout of $\left(\mathbf{A}, \mathbf{B}, \mathbf{C}, f_{B}, f_{C}\right)$ and the pushout maps are $\mathrm{E}\left(\pi_{B}\right) \circ e_{\mathbf{B}}: \mathbf{B} \rightarrow \mathrm{E}(\mathbf{X})$ and $\mathrm{E}\left(\pi_{C}\right) \circ e_{\mathbf{C}}: \mathbf{C} \rightarrow \mathrm{E}(\mathbf{X})$. Since $\mathrm{E}\left(\pi_{B}\right) \circ e_{\mathbf{B}}$ and $\mathrm{E}\left(\pi_{C}\right) \circ e_{\mathbf{C}}$ are embeddings, the $V$-formation $\left(\mathbf{A}, \mathbf{B}, \mathbf{C}, f_{B}, f_{C}\right)$ admits amalgamation.

As we mentioned in Section 1, in [18] Maksimova proved that $\mathcal{G}_{n}$ fails to satisfy the amalgamation property if $n \geqslant 4$. Combining this with Lemma 6.1 , we obtain the following.

Corollary 6.2. The variety $\mathcal{G}_{n}$, with $n \geqslant 4$, does not admit a total (single-sorted) strong duality.

Given a natural duality, the problem of describing which maps between dual structures correspond to embeddings between algebras is not as simple in general as it is when the duality is strong and based on a total structure. However for the Gödel algebra varieties $\mathcal{G}_{n}$ the task of describing such maps is greatly facilitated by two features which work to our advantage. Firstly, we can call on the two-way translation between our natural dualities and the Priestley/Esakia duality. Secondly, the latter duality is the restriction to Heyting algebras of Priestley duality, which is strong and has a total structure as the alter ego.

Lemma 6.3. Let $\mathbf{A}, \mathbf{B} \in \mathcal{G}_{n}$ with $n \geqslant 2$ and $f: \mathbf{A} \rightarrow \mathbf{B}$ be a homomorphism. For each $\sigma \in \Sigma_{n}$, the following statements are equivalent:

(1) $f$ is an embedding;

(2) for each $x \in \mathrm{D}^{\sigma}(\mathbf{A})$, there exists $y \in \mathrm{D}^{\sigma}(\mathbf{B})$ such that $\mathrm{D}^{\sigma}(f)(y) \simeq_{\mathrm{D}^{\sigma}(\mathbf{A})}^{\sigma} x$.

Proof. The map $f$ is an embedding if and only if $\mathrm{HU}(f)$ is surjective. Since the functor $\mathrm{HU}$ is naturally isomorphic to $\mathrm{F}^{\sigma} \mathrm{D}^{\sigma}$, the map $\mathrm{HU}(f)$ is surjective if and only if $\mathrm{F}^{\sigma}\left(\mathrm{D}^{\sigma}(f)\right)$ is surjective. 
From the observations we made about the functor $\mathrm{F}^{\sigma}$ in Section 4 we have $\mathrm{F}^{\sigma}\left(\mathrm{D}^{\sigma}(f)\right)\left([z]_{\simeq_{\mathrm{D}^{\sigma}(\mathbf{B})}}\right)=$ $\left[\mathrm{D}^{\sigma}(f)(z)\right]_{\simeq_{\mathrm{D}^{\sigma}(\mathbf{A})}^{\sigma}}$. Therefore $\mathrm{F}^{\sigma}\left(\mathrm{D}^{\sigma}(f)\right)$ is surjective if and only if $(2)$ holds.

We are now ready to prove the main result of this section.

Theorem 6.4. Let $\left(\mathbf{A}, \mathbf{B}, \mathbf{C}, f_{B}, f_{C}\right)$ be a $V$-formation in $\mathcal{G}_{n}$ with $n \geqslant 4$. Let $\sigma=\left(g_{1}, \ldots, g_{n-3}, h_{1}\right)$. Then the following statements are equivalent:

(1) $\left(\mathbf{A}, \mathbf{B}, \mathbf{C}, f_{B}, f_{C}\right)$ admits amalgamation;

(2) the dual maps $\mathrm{D}^{\sigma}\left(f_{B}\right)$ and $\mathrm{D}^{\sigma}\left(f_{C}\right)$ satisfy the following conditions:

(a) for each $x \in \mathrm{D}^{\sigma}(\mathbf{B})$, there exist $y \in \mathrm{D}^{\sigma}(\mathbf{B})$ and $z \in \mathrm{D}^{\sigma}(\mathbf{C})$ such that $x \simeq_{\mathrm{D}^{\sigma}(\mathbf{B})}^{\sigma} y$ and $\mathrm{D}^{\sigma}\left(f_{B}\right)(y)=\mathrm{D}^{\sigma}\left(f_{B}\right)(z) ;$ and

(b) for each $x^{\prime} \in \mathrm{D}^{\sigma}(\mathbf{C})$, there exist $y^{\prime} \in \mathrm{D}^{\sigma}(\mathbf{C})$ and $z^{\prime} \in \mathrm{D}^{\sigma}(\mathbf{B})$ such that $x^{\prime} \simeq_{\mathrm{D}^{\sigma}(\mathbf{C})}^{\sigma} y^{\prime}$ and $\mathrm{D}^{\sigma}\left(f_{C}\right)\left(y^{\prime}\right)=\mathrm{D}^{\sigma}\left(f_{B}\right)\left(z^{\prime}\right)$.

Proof. In this proof we only consider $\sigma=\left(g_{1}, \ldots, g_{n-3}, h_{1}\right)$, therefore we shall omit the superscripts and write $\mathbf{C}_{n}, \mathrm{D}$ and $\mathrm{E}$ instead of $\mathbf{C}_{n}^{\sigma}, \mathrm{D}^{\sigma}$ and $\mathrm{E}^{\sigma}$, respectively.

As in Lemma 6.1, let $\mathbf{X}$ be the subspace of $\mathrm{D}(\mathbf{B}) \times \mathrm{D}(\mathbf{C})$ whose universe is $\{(x, y) \in \mathrm{D}(\mathbf{B}) \times$ $\left.\mathrm{D}(\mathbf{C}) \mid \mathrm{D}\left(f_{B}\right)(x)=\mathrm{D}\left(f_{C}\right)(y)\right\}$, that is, the fibred product $\mathrm{D}(\mathbf{B}) \stackrel{\mathrm{D}\left(f_{B}\right)}{\longrightarrow} \mathrm{D}(\mathbf{A}) \stackrel{\mathrm{D}\left(f_{C}\right)}{\longleftarrow} \mathrm{D}(\mathbf{C})$. By Theorem 5.1 , the duality determined by $\mathbf{C}_{n}$ is full. Therefore $\mathbf{E}(\mathbf{X})$ is the pushout of $\left(\mathbf{A}, \mathbf{B}, \mathbf{C}, f_{B}, f_{C}\right)$ with pushout maps $\mathrm{E}\left(\pi_{B}\right) \circ e_{\mathbf{B}}: \mathbf{B} \rightarrow \widetilde{\mathrm{E}}(\mathbf{X})$ and $\mathrm{E}\left(\pi_{C}\right) \circ e_{\mathbf{C}}: \mathbf{C} \rightarrow \mathrm{E}(\mathbf{X})$. Now observe that $\pi_{B}$ satisfies condition (2) in Lemma 6.3 if and only if (a) holds. This proves that $\mathrm{E}\left(\pi_{B}\right)$ is an embedding if and only if (a) holds. Similarly, $\mathrm{E}\left(\pi_{C}\right)$ is an embedding if and only if (b) holds.

Our last application concerns coproducts of Gödel algebras. The coproduct of a family of algebras depends not only on the algebras involved but also on the class in which we form the coproduct. In particular, given a set $\mathcal{K}$ of algebras in $\mathcal{G}_{n} \subseteq \mathcal{G}$, their coproduct in $\mathcal{G}$ might be different from their coproduct in $\mathcal{G}_{n}$. Nonetheless, if the set $\mathcal{K}$ is finite, the coproduct formed in $\mathcal{G}$ coincides with the coproduct formed in $\mathcal{G}_{m}$, for a suitably large $m$; in particular, it is enough to consider $m=2+\sum_{\mathbf{A} \in \mathcal{K}}\left(k_{\mathbf{A}}-2\right)$, where $k_{\mathbf{A}}$ is the minimal $k$ such that $\mathbf{A} \in \boldsymbol{G}_{k}$ for all $\mathbf{A} \in \mathcal{K}$. These observations give us access to certain coproducts in $\mathcal{G}$. In particular we can calculate coproducts in $\mathcal{G}$ of any finite set of algebras belonging to $\bigcup_{n} \mathcal{G}_{n}$.

Natural dualities, when available, are a good tool for the study of coproducts: under such a duality, the dual space of the coproduct of a family of algebras corresponds to the cartesian product of their dual spaces. This fact was already noted in [7, Section 5], where it was applied to determine finitely generated free algebras in $\mathcal{G}_{n}$ and in $\mathcal{G}$, with the calculations performed wholly within the natural duality setting. The main difficulty one encounters in attempting to use a natural duality to calculate the coproduct in $\mathcal{G}_{n}$ or in $\mathcal{G}$ of a family of algebras $\mathcal{K} \subseteq \mathcal{G}_{n}$ lies in finding effective descriptions of the dual spaces of the algebras $\mathbf{A} \in \mathcal{K}$ and then of the algebra dual to the resulting cartesian product.

On the other hand, using Priestley/Esakia duality instead, as D'Antona and Marra do in [6], brings different challenges. While dual spaces may be easy to visualise, the duality functor (viz. the restriction of $\mathrm{HU}$ to $\boldsymbol{\mathcal { G }}_{n}$; see Fig. 2) does not in general convert a coproduct in $\boldsymbol{\mathcal { S }}_{n}$ to a cartesian product, so that the dual space of a coproduct is hard to describe. D'Antona and Marra proceed in the following way to describe the coproduct of any finite family $\mathcal{K}$ of finite (equivalently, finitely generated) Gödel algebras. They first find the Priestley/Esakia dual of each algebra in $\mathcal{K}$. Then, using the fact that product distributes over coproduct in the category of Esakia spaces (see [11, p. 391], where it is proved that, in every variety of Heyting algebras, coproduct distributes over product), the problem reduces to describing the product of two finite trees in a suitable category. In bare outline, the authors' general strategy to construct the product of two trees is: first to represent each tree by a family of ordered partitions; second, to construct another family of ordered partitions from the original ones by suitably shuffling and merging these. Finally they must reverse the process to obtain a tree from the resulting set of ordered partitions.

Our two-way translation allows us to work with a natural duality and Priestley/Esakia in combination, and so gives a simpler approach than one based on either of these duality techniques alone. We present our method as a 5 -step procedure. We shall fix $\sigma \in \Sigma_{n}$ to be $\left(g_{1}, \ldots, g_{n-3}, h_{n-2}\right)$ 


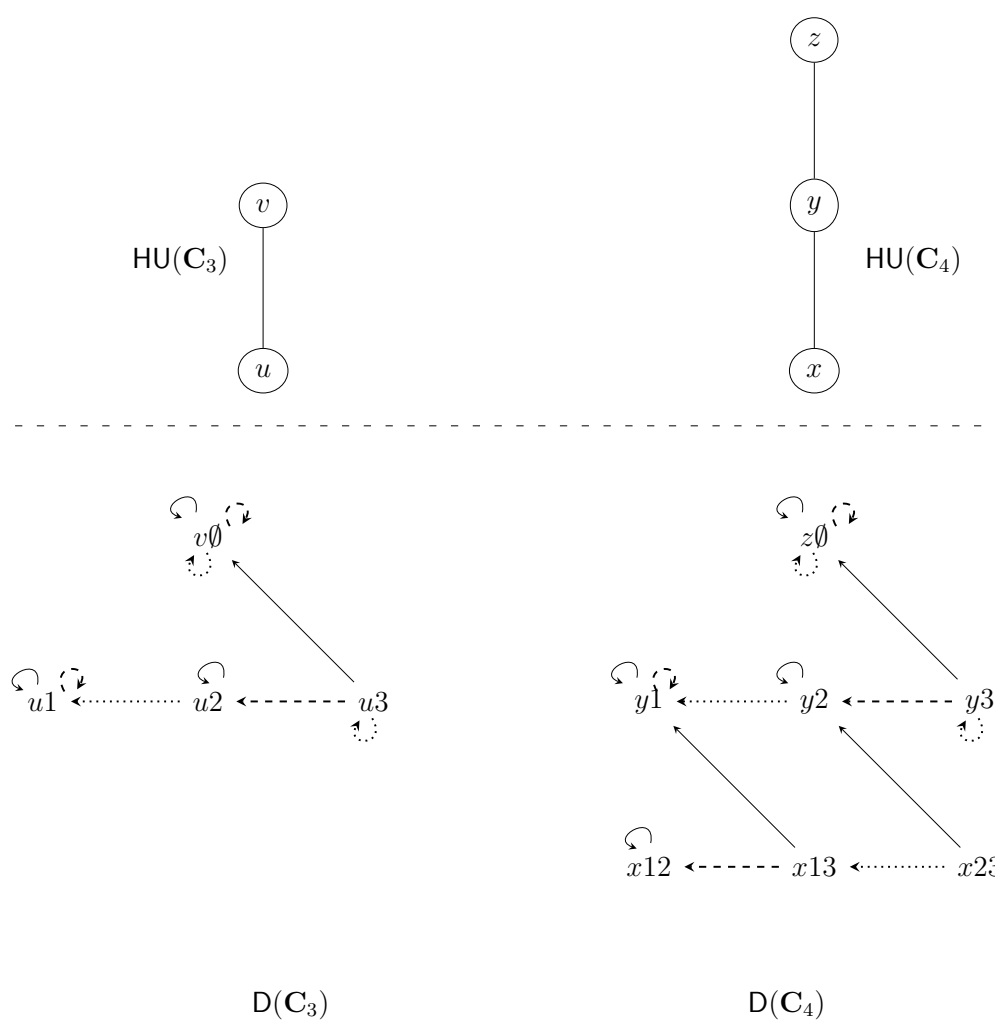

Figure 6. Calculating $\mathbf{C}_{3} \coprod_{\mathcal{G}_{5}} \mathbf{C}_{4}$ : Steps 1 and 2

because this gives particularly simple pictures in examples, but any other choice of $\sigma$ would also be legitimate. To calculate $\coprod_{\mathfrak{S}_{n}} \mathcal{K}$, where $\mathcal{K} \subseteq \mathcal{G}_{n}$ :

1. For each $\mathbf{A} \in \mathcal{K}$, determine $\mathrm{HU}(\mathbf{A})$.

2. Use Theorem 4.6 to construct $\mathrm{G}^{\sigma} \mathrm{HU}(\mathbf{A})$ from $\mathrm{HU}(\mathbf{A})$, for each $\mathbf{A} \in \mathcal{K}$.

3. Form the cartesian product $\mathbf{X}=\prod\left\{\mathrm{G}^{\sigma} \mathrm{HU}(\mathbf{A}) \mid \mathbf{A} \in \mathcal{K}\right\}$.

4. Determine $\simeq_{\mathbf{x}}^{\sigma}, \prec_{\mathbf{x}}^{\sigma}$ and $\left(\mathbf{X} / \simeq_{\mathbf{x}}^{\sigma} ; \leqslant_{\mathbf{x}}^{\sigma}, \mathcal{T}^{\mathbf{x}} / \simeq_{\mathbf{x}}^{\sigma}\right)$ as in Theorem 4.3 .

5. $\operatorname{VK}\left(\mathbf{X} / \simeq_{\mathbf{x}}^{\sigma}, \leqslant_{\mathrm{x}}^{\sigma}, \mathcal{T}^{\mathrm{x}} / \simeq_{\mathrm{x}}^{\sigma}\right)$ gives $\coprod_{\mathcal{S}_{n}} \mathcal{K}$.

Of course the procedure described above does not constitute an algorithm unless restricted to a finite family of finite algebras.

Some comments on our strategy as compared with that in [6] should be made. Our procedure allows us on the one hand to be flexible and to calculate coproducts in any subvariety $\mathcal{G}_{k}$ that contains the algebras, and on the other hand, most significantly, we replace the involved procedure of calculating shuffles and merges simply by the computation of a cartesian product of structured spaces. It might be seen as a disadvantage that we need to know at the outset in which Gódel subvariety $\mathcal{G}_{n}$ we need to work in order that the coproduct calculated in $\mathcal{G}_{n}$ coincides with that calculated in $\mathcal{G}$. But this is a minor issue: since, as we observed before, a suitable $n$ can easily be found once the finite family of algebras is fixed. From the perspective of the procedure developed in [6], one can similarly cut down to a finitely generated subvariety $\mathcal{G}_{k}$. However to do so one needs first to calculate the Priestley/Esakia dual of the coproduct in $\mathcal{G}$ and then to truncate the resulting forest to obtain a forest of depth $k-2$. 


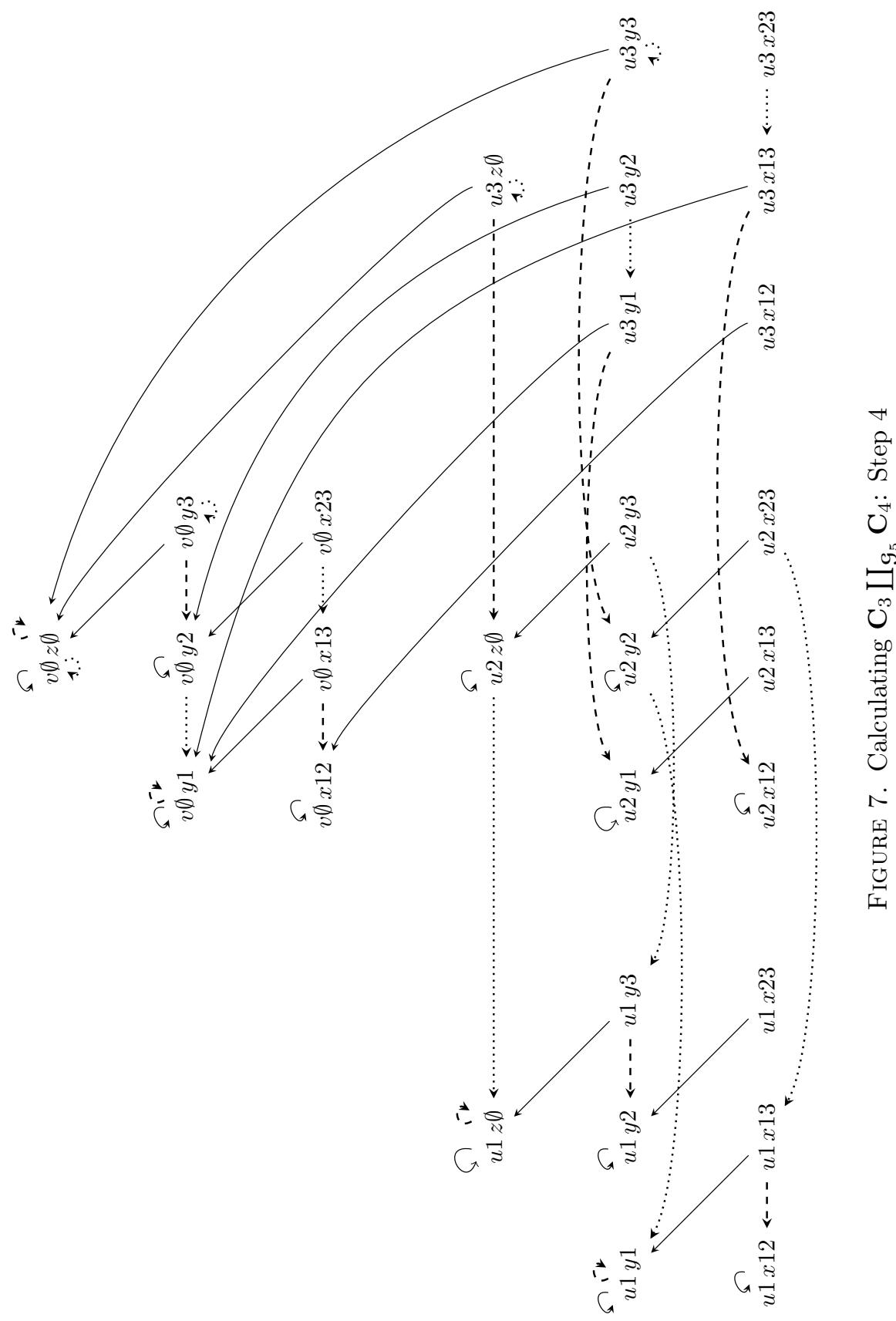

We now illustrate how our method works in practice. The first example we choose is the one given in [6, Examples 1 and 2]. This is done to enable the reader to compare and contrast the two procedures. (Observe that in [6] the order in the Priestley/Esakia spaces is the dual of the one considered in this paper.)

Example 6.5. Let us determine $\mathbf{C}_{3} \amalg_{\mathcal{G}} \mathbf{C}_{4}$. First observe $\mathbf{C}_{3} \amalg_{\mathcal{G}} \mathbf{C}_{4}$ belongs to $\mathcal{G}_{5}$. We take $\sigma=\left(g_{1}, g_{2}, h_{3}\right)$.

Step 1: Let $\mathrm{HU}\left(\mathbf{C}_{3}\right)=(\{u, v\} ; \leqslant)$ where $u<v$ and $\mathrm{HU}\left(\mathbf{C}_{3}\right)=(\{x, y, z\} ; \leqslant)$ with $x<y<z$ (see Fig. 6).

Step 2: Figure 6 also shows $\mathrm{G}^{\sigma}\left(\mathrm{HU}\left(\mathbf{C}_{3}\right)\right)$ and $\mathrm{G}^{\sigma}\left(\mathrm{HU}\left(\mathbf{C}_{4}\right)\right)$. 


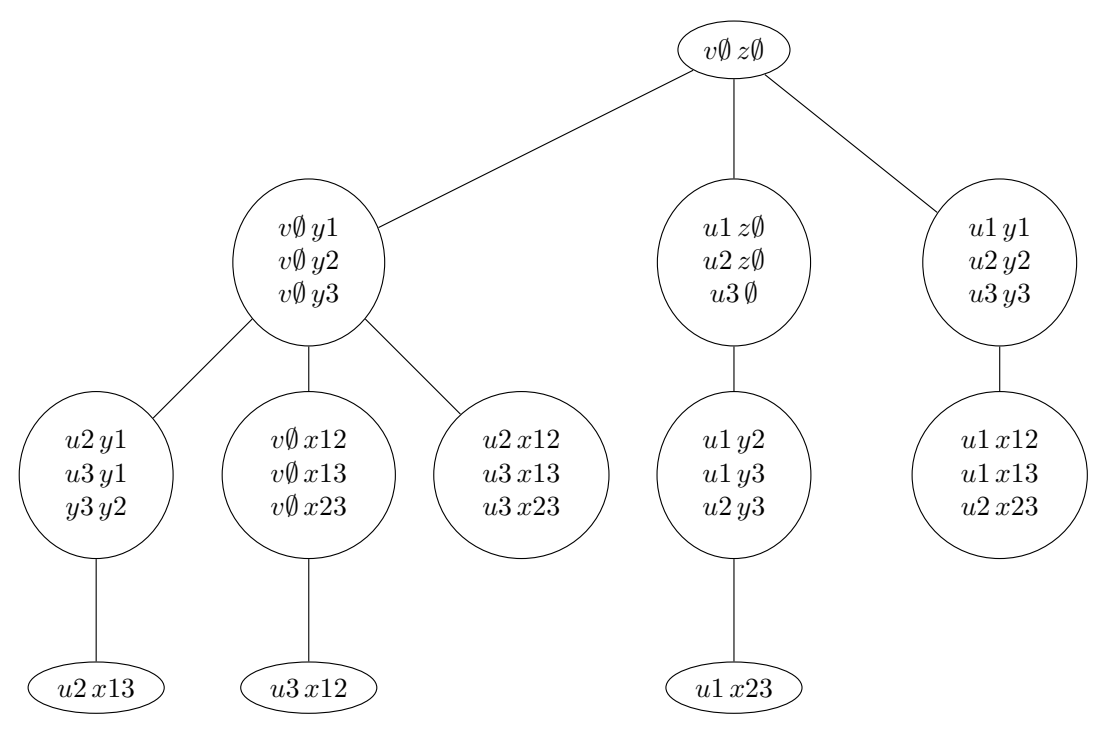

Figure 8. Calculating $\mathbf{C}_{3} \amalg_{\mathcal{G}_{5}} \mathbf{C}_{4}$ : Step 4
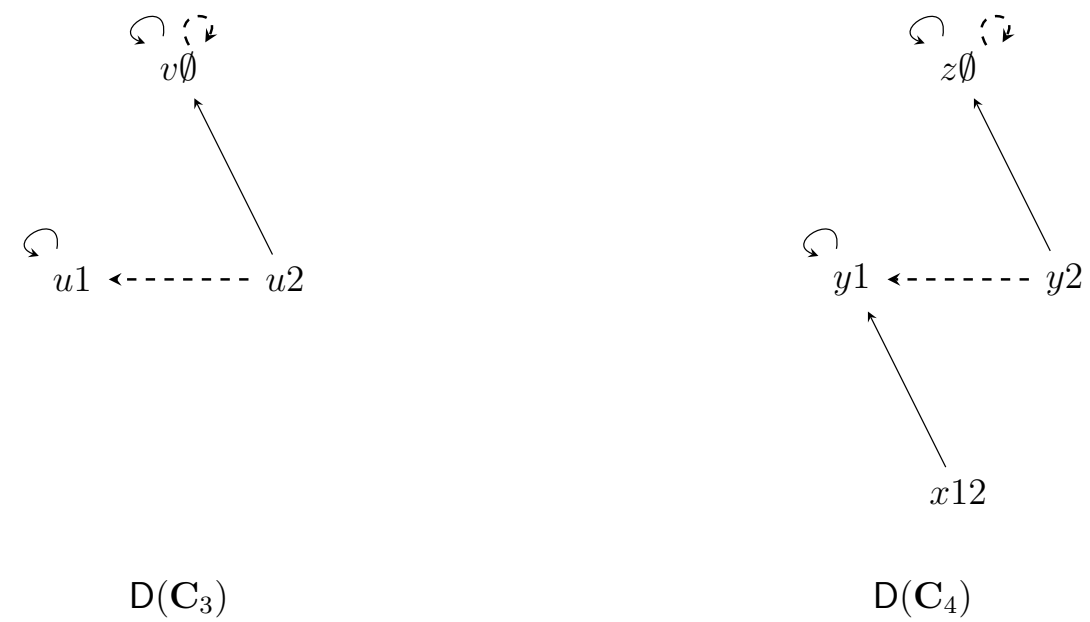

$\mathrm{D}\left(\mathbf{C}_{3}\right)$

$\mathrm{D}\left(\mathrm{C}_{4}\right)$

Figure 9. Calculating $\mathbf{C}_{3} \coprod_{\mathcal{G}_{4}} \mathbf{C}_{4}$ : Step 2

Step 3: The cartesian product $\mathrm{G}^{\sigma}\left(\mathrm{HU}\left(\mathbf{C}_{3}\right)\right) \times \mathrm{G}^{\sigma}\left(\mathrm{HU}\left(\mathbf{C}_{3}\right)\right)$ is represented in Fig. 7 .

Step 4: Figure 8 depicts the calculation of $\mathrm{F}^{\sigma}\left(\mathrm{G}^{\sigma}\left(\mathrm{HU}\left(\mathbf{C}_{3}\right) \times \mathrm{G}^{\sigma}\left(\mathrm{HU}\left(\mathbf{C}_{4}\right)\right)\right.\right.$ and therefore isomorphic to $\mathrm{HU}\left(\mathbf{C}_{3} \coprod_{\mathbf{G}_{5}} \mathbf{C}_{4}\right)$.

Step 5: The lattice whose Priestley dual is shown in Fig. 8 is (the reduct of the Gödel algebra) isomorphic to $\perp \oplus\left(\left(\perp \oplus\left(\mathbf{C}_{3} \times \mathbf{C}_{3} \times \mathbf{C}_{2}\right)\right) \times \mathbf{C}_{4} \times \mathbf{C}_{3}\right)$, where $\oplus$ denotes linear sum.

Example 6.6. Since $\mathbf{C}_{3}$ and $\mathbf{C}_{4}$ lie in $\mathcal{G}_{4}$, we can also calculate $\mathbf{C}_{3} \coprod_{\mathfrak{g}_{4}} \mathbf{C}_{4}$. Here Step 1 is the same as in Example 6.5. Steps 2-4 are shown in Figs. 9-11. We see that the tree $\mathbf{X}=$ $\mathrm{G}^{\sigma}\left(\mathrm{F}^{\sigma} \mathrm{HU}\left(\mathbf{C}_{3}\right) \times \mathrm{F}^{\sigma} \mathrm{HU}\left(\mathbf{C}_{4}\right)\right)$ is (isomorphic to) a truncation of the one obtained in Step 4 of Example 6.5. It is only for $n \geqslant 5$ that the coproduct in $\mathcal{G}_{n}$ coincides with that in $\mathcal{G}$. Finally, to 


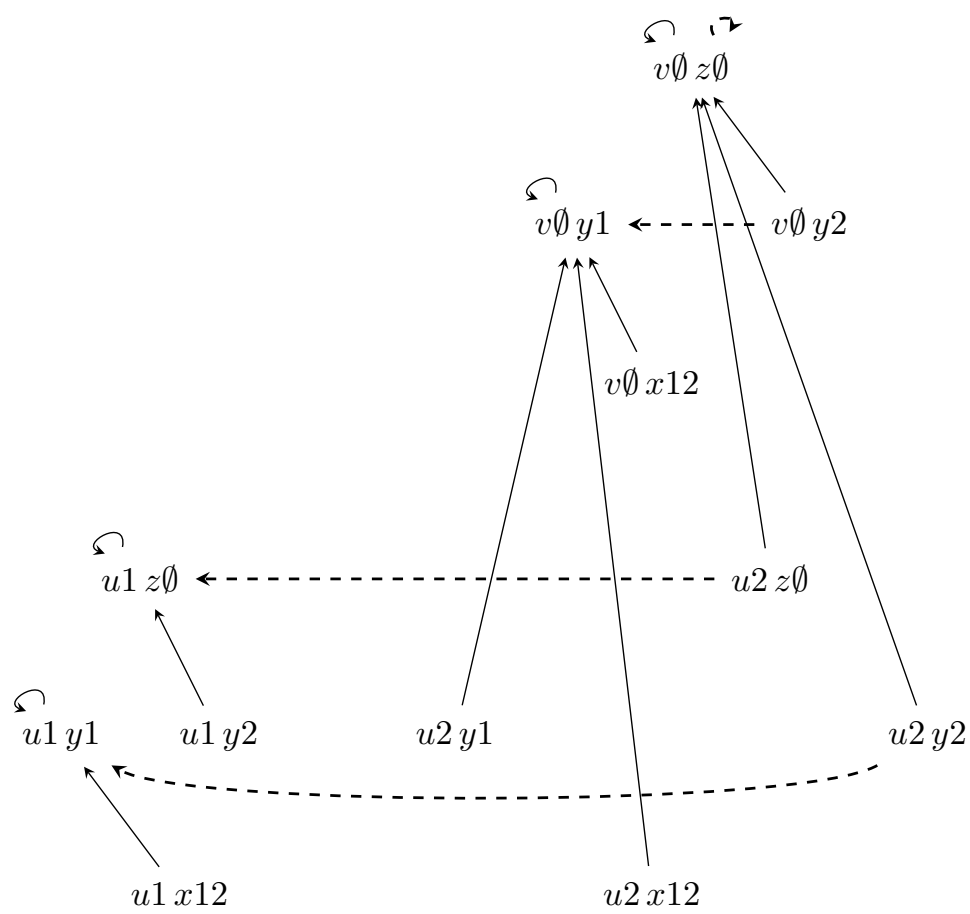

Figure 10. Calculating $\mathbf{C}_{3} \amalg_{\mathfrak{G}_{4}} \mathbf{C}_{4}$ : Step 3

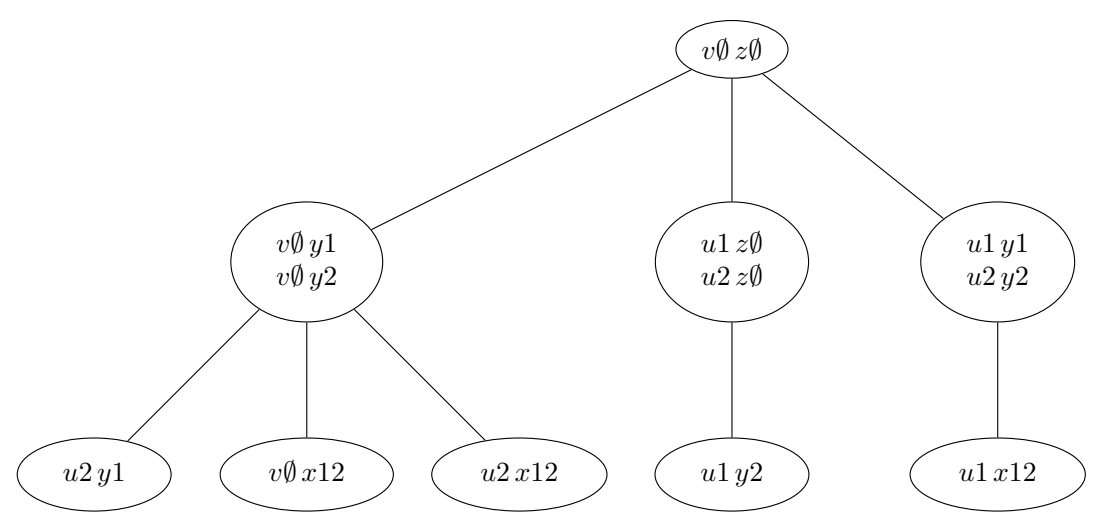

Figure 11. Calculating $\mathbf{C}_{3} \amalg_{\mathcal{G}_{4}} \mathbf{C}_{4}$ : Step 4

complete Step 5 we observe that the dual lattice of $\mathbf{X}$ is

$$
\perp \oplus\left(\left(\perp \oplus\left(\mathbf{C}_{2} \times \mathbf{C}_{2} \times \mathbf{C}_{2}\right)\right) \times \mathbf{C}_{3} \times \mathbf{C}_{3}\right),
$$

which has 82 elements.

\section{REFERENCES}

[1] R. Balbes and Ph. Dwinger, Distributive Lattices. Missouri University Press (1974)

[2] G. Bezhanishvili, N. Bezhanishvili, D. Gabelaia and A. Kurz, Bitopological dualities for distributive lattices and Heyting algebras. Math. Struct. Comput. Sci. 20, 359-393 (2010) 
[3] L.M. Cabrer and H.A. Priestley, Coproducts of distributive lattice-based algebras. Algebra Universalis (to appear), arxiv: 1308.4650

[4] D.M. Clark and B.A. Davey, Dualities for the Working Algebraist. Cambridge University Press (1998)

[5] J. Czelakowski and D. Pigozzi, Amalgamation and interpolation in abstract algebraic logic. In Models, Algebras, and Proofs (eds. X. Caicedo and C.H. Montenegro), Lecture Notes in Pure and Appl. Mathematics Vol. 203, 187-265 (1999)

[6] O.M. D'Antona and V. Marra, Computing coproducts of finitely presented Gödel algebras. Ann. Pure Appl. Logic 142, 202-211 (2006)

[7] B.A. Davey, Dualities for equational classes of Brouwerian algebras and Heyting algebras. Trans. Amer. Math. Soc. 221, 119-146 (1976)

[8] B.A. Davey, M. Haviar and T. Niven, When is a full duality strong?, Houston J. Math. (electronic) 33, 1-22 (2007)

[9] B.A. Davey and M.R. Talukder, Dual categories for endodualisable Heyting algebras: optimization and axiomatization. Algebra Universalis 53, 331-355 (2005)

[10] B.A. Davey and H. Werner, Piggyback-Dualitäten. Bull. Austral. Math. Soc. 32, 1-32 (1985)

[11] B.A. Davey and H. Werner, Distributivity of coproducts over products. Algebra Universalis 12, 387-394 (1981)

[12] B.A. Davey and H.A. Priestley, Introduction to Lattices and Order, 2nd edn. Cambridge University Press (2002)

[13] M.A.E. Dummett, A propositional calculus with a denumerable matrix. J. Symb. Logic 24, 96-107 (1959)

[14] L.L. Esakia, Topological Kripke models. Soviet Math. Dokl. 15, 147-151 (1974)

[15] K. Gödel, Zum intuitionistischen Aussagenkalkül. Anz. Akad. Wiss. Wien 69, 65-66 (1932). Reprinted in Gödel's collected Works Vol.1, Oxford University Press (1986), pp. 222-225 added

[16] T. Hecht and T. Katriňák, Equational classes of relative Stone algebras. Notre Dame J. Formal Logic 13, 248-254 (1972)

[17] A. Horn, Free L-algebras. J. Symb. Logic 34, 475-480 (1969)

[18] L.L. Maksimova, Craig's theorem in superintuitionistic logics and amalgamable varieties of pseudoboolean algebras, Algebra i Logika 16, 643-681 (1977)

[19] S. Mac Lane, Categories for the Working Mathematician. Grad. Texts in Math. Vol. 5, Springer-Verlag (1969)

[20] J. von Plato, Skolem's discovery of Gödel-Dummett logic. Studia Logica 73, 153-157 (2003)

E-mail address: 1.cabrer@disia.unifi.it

(lmc) Dipartimento di Statistica, Informatica, Applicazioni, Università degli Studi di Firenze, 59 Viale Morgani, 50134, Italy

E-mail address: hap@maths.ox.ac.uk

Mathematical Institute, University of Oxford, Radcliffe Observatory Quarter, Oxford OX2 6GG, UNITED KingDOM 